Sharif University of Technology
Scientia Iranica
Transactions E: Industrial Engineering
wCIENTIA

\title{
Optimal multi-product dynamic pricing and inventory policies for coordinating production and marketing under fuzzy environment: Fuzzy expansion methods
}

\author{
B. Vahdani ${ }^{a, *}$,Sh. Sadigh Behzadi ${ }^{b}$ and T. Allahviranloo ${ }^{c}$ \\ a. Faculty of Industrial and Mechanical Engineering, Qazvin Branch, Islamic Azad University, Qazvin, P.O. Box 3419759811, Iran. \\ b. Department of Mathematics, Qazvin Branch, Islamic Azad University, Qazvin, P.O. Box 3419759811, Iran. \\ c. Department of Mathematics, Science and Research Branch, Islamic Azad University, Tehran, P.O. Box 14515/775, Iran.
}

Received 24 August 2014; received in revised form 21 July 2015; accepted 15 September 2015

\section{KEYWORDS}

Dynamic programming;

Pricing-inventory policies;

Uncertainty;

Fuzzy expansion method.

\begin{abstract}
This paper investigates the optimal multi-product dynamic pricing and inventory policies over a multi-period planning horizon with deteriorating products and a fuzzy demand function. The objective is maximization of the discount profit. A dynamic programming model is presented to determine retail price and replenishment quantities. Also, due to the existence of uncertainties in the values of parameters, such as cost, deterioration rate, and the optimal strategies in general, they cannot be obtained with high feasibility. Thus, the concept of fuzzy set theory can be applied to cope whit this issue. Since the presented model is a fuzzy partial deferential equation, three novel fuzzy expansion methods, including Jacobi polynomials, airfoil polynomials, and fuzzy collocation methods, are proposed for solving this problem. Finally, this paper carries out various computational experiments to assess the proposed model and solution approaches.

(C) 2016 Sharif University of Technology. All rights reserved.
\end{abstract}

\section{Introduction}

Nowadays, with respect to the increase of competition among companies, the performance of the companies can be enhanced by employing different marketing policies for different product groups. One of the most effective policies is coordination of dynamic pricing and inventory management, since using this policy makes it possible to take the appropriate decision about the production, planning, and inventory. Hence, unnecessary costs can be prevented, thus, increasing profits [1]. Therefore, since the maximum profit is the main goal during the planning horizon of any organization, integration and cooperation of production and marketing policies to achieve the maximum profit are the main priorities of each organization. In

\footnotetext{
*. Corresponding author.

E-mail address: b.vahdani@gmail.com (B. Vahdani)
}

recent years, many researchers have increasingly placed emphasis on integrating production and inventory policies. But, in many of these research works, demand function and parameters are certain and deterministic. However, in many real-world problems, achieving the desired data is not possible [2]. In order to consider the uncertainty in the parameters and the data for this category of problems, the use of the concept of fuzzy sets is an appropriate approach, which is introduced by Negoita et al. [3]. On the other hand, in a significant number of these research works, in which parameters and data are considered to be fuzzy sets, the values obtained for the objective function and decision variables are deterministic, while it is reasonable that, in a fuzzy environment, a fuzzy result should be made to meet the assumptions. However, in a significant number of these research works, time is assumed to be discrete. Moreover, the price of the product can change in these fixed points. This paper investigates the 
optimal multi-product dynamic pricing and inventory policies over a multi-period planning horizon with deteriorating products and a fuzzy demand function and fuzzy parameters. For this purpose, a fuzzy dynamic programming model is presented to determine retail price and replenishment quantities. Since the presented model is a fuzzy deferential equation, three novel fuzzy expansion methods including Jacobi polynomials, airfoil polynomials, and fuzzy collocation methods for solving this problem are proposed. Our contributions are twofold. First, a fuzzy multi-product dynamic pricing and inventory policies over a multiperiod planning horizon with deteriorating products in continuous time are proposed. Second, to the best of our knowledge, this is the first effort that uses the fuzzy expansion methods for solving problems in the field of pricing and inventory management. Hence, we proposed three novel fuzzy expansion methods including Jacobi polynomials, airfoil polynomials, and fuzzy collocation methods for solving the proposed model. The remainder of the paper is organized as follows. Section 2 presents a brief review of the literature. Some basic definitions are described in Section 3. Problem definition and formulation are described in Section 4. The proposed fuzzy expansion methods are given in Section 5. Computational experiments are provided in Section 6. Finally, the paper is concluded in Section 7.

\section{Literature review}

Deterministic models and fuzzy model and the approaches are discussed here. Numerous research works have been carried out in relation to the deterministic models and approaches. Jrgensen and Kort [4] investigated an optimal control model of pricing and inventory strategy in a multi-stage system. Gupta et al. [5] studied a discrete-time model with considering timedependent reservation prices of clients in deterministic environment. Jung and Klein [6] considered three inventory problems with respect to different functions of cost. They utilized a geometric programming approach to obtain selling price and order quantity. Pal et al. [7] considered a partially integrated production and marketing policy model for obtaining selling price, production rate demand, marketing expenditure, and the length of a product's life cycle. They assumed that the planning was done for a product. Moreover, they proposed a generalized reduced gradient method and simple genetic algorithm for the proposed model. Transchel and Minner [8] studied the impact of the relation between dynamic pricing and inventory control on single product economic order decision. Pang [1] investigated an inventory control system and dynamic pricing policies over a multi-period system. The unmet demand of customers might be partially backlogged. Moreover, the inventory might deteriorate in the plan- ning horizon. Herbon et al. [9] proposed a non-linear mixed-integer mathematical programming model for investigating an inventory control system, considering a perishing product with price- and freshness-dependent consumption. They also considered exponential deterioration for the product price and life cycle of products. Moreover, they proposed a local search algorithm for solving the proposed model. Chen [10] proposed an optimal control model of pricing and inventory strategy in a vertically decentralized supply chain over a multiperiod time horizon. The objective function considered in this research was the maximization of the discount profit. Moreover, the author considered two options for applying business, namely Retailer Managed Inventory (RMI) and Vendor Managed Inventory (VMI). Regarding the fuzzy models and approaches, little research has been carried out. Liu [11] presented an optimal model of pricing and inventory strategy with price-dependent demand and order-quantity-dependent unit cost in the fuzzy environment. Moreover, he proposed a solution approach based on Geometric Programming (GM) approach for solving the proposed model. Sadjadi et al. [12] developed a pricing and inventory model for marketing planning in the fuzzy environment. Also, they proposed a solution methodology based on GM approach for solving the proposed model. Samadi et al. [2] presented a fuzzy inventory-marketing model with shortages. They formulated their problem as GM approach. Soni and Joshi [13] presented a simple inventory model in the fuzzy environment for coordinating pricing and inventory strategies in a supply chain. They formulated their problem utilizing a trade credit policy approach, in which the provider granted the retailer a permissible delay period and the retailer, sequentially, offered providers a permissible delay period. Cosgun et al. [14] proposed an IFTHEN-rule based approach in the fuzzy environment for dynamic pricing problem. The objective was to find the optimal prices utilizing fuzzy possibilistic programming and dynamic programming approaches. Zhao and Wang [15] studied the retail service decisions and pricing in a supply chain. They considered one manufacturer and two retailers. Also, demands of customers, manufacturing costs, and service costs were fuzzy. Moreover, they presented three different game structures including manufacturer-leader stackelberg, retailer-leader Stackelberg, and vertical Nash. Sadeghi and Akhavan Niaki [16] proposed a bi-objective vendor managed inventory model for a supply chain problem. They considered single vendor and multiple retailers. Also, the customers' demand was fuzzy. The two objectives that had to be optimized were the minimization of the total inventory cost and the minimization of the warehouse space. Moreover, they proposed two multi-objective evolutionary algorithms for solving this problem. 


\section{Basic definitions}

In this section, we represent some basic definitions of a fuzzy set and its operations.

Definition 1. An arbitrary fuzzy number, $\widetilde{u}$, is denoted by an ordered pair of functions $(\underline{u}, \bar{u})$ that satisfy the following three conditions $[17,18]$ :

(i) $\bar{u}: r \rightarrow u_{r}^{-} \in \mathbb{R}$ is a bounded left-continuous non-decreasing function over $[0,1]$;

(ii) $\underline{u}: r \rightarrow u_{r}^{+} \in \mathbb{R}$ is a bounded left-continuous non-increasing function over $[0,1]$;

(iii) $\underline{u} \leq \bar{u}, 0 \leq r \leq 1$.

Definition 2. For arbitrary fuzzy numbers, we utilize the Hausdorff distance [17] where $(E, D)$ is a complete metric space and the properties are as follows:

$$
\begin{aligned}
& D(\widetilde{u} \oplus \widetilde{w}, \widetilde{v} \oplus \widetilde{w})=D(\widetilde{u}, \widetilde{v}), \quad \forall \widetilde{u}, \widetilde{v} \in E, \\
& D(k \widetilde{u}, k \widetilde{v})=|k| D(\widetilde{u}, \widetilde{v}), \quad \forall k \in \mathbb{R}, \widetilde{u}, \widetilde{v} \in E, \\
& D(\widetilde{u} \oplus \widetilde{v}, \widetilde{w} \oplus \widetilde{e}) \leq D(\widetilde{u}, \widetilde{w})+D(\widetilde{v}, \widetilde{e}),
\end{aligned}
$$

$\forall \widetilde{u}, \widetilde{v}, \widetilde{w}, \widetilde{e} \in E$

Definition 3. Consider $\widetilde{x}, \widetilde{y} \in E$. If there exists $\widetilde{z} \in$ $E$ such that $\widetilde{x}=\widetilde{y} \oplus \widetilde{z}$ then $\widetilde{z}$ is called the $H$-difference of $\widetilde{x}$ and $\widetilde{y}$, and is denoted by $\widetilde{x} \ominus \widetilde{y}[15]$.

Definition 4. Let $\tilde{f}:(a, b) \rightarrow E$ and $x_{0} \in(a, b)$. We say that $f$ is generalized differentiable at $x_{0}$ (Bede-Gal differentiability) if there exists an element $\tilde{f}^{\prime}\left(x_{0}\right) \in E$, such that [17]:

(i) For all sufficiently small $h>0, \exists \widetilde{f}\left(x_{0}+h\right) \ominus$ $\widetilde{f}\left(x_{0}\right), \exists \widetilde{f}\left(x_{0}\right) \ominus \widetilde{f}\left(x_{0}-h\right)$ and the limits, we have the following relation:

$$
\begin{aligned}
& \lim _{h \rightarrow 0} \frac{\tilde{f}\left(x_{0}+h\right) \ominus \tilde{f}\left(x_{0}\right)}{h} \\
& \quad=\lim _{h \rightarrow 0} \frac{\tilde{f}\left(x_{0}\right) \ominus \tilde{f}\left(x_{0}-h\right)}{h}=\tilde{f}^{\prime}\left(x_{0}\right),
\end{aligned}
$$

or:

(ii) For all sufficiently small $h>0, \exists \tilde{f}\left(x_{0}\right) \ominus \tilde{f}\left(x_{0}+\right.$ $h), \exists \tilde{f}\left(x_{0}-h\right) \ominus \tilde{f}\left(x_{0}\right)$ and the limits, we have the following relation:

$$
\begin{aligned}
\lim _{h \rightarrow 0} \frac{\tilde{f}\left(x_{0}\right) \ominus \tilde{f}\left(x_{0}+h\right)}{-h} \\
\quad=\lim _{h \rightarrow 0} \frac{\tilde{f}\left(x_{0}-h\right) \ominus \tilde{f}\left(x_{0}\right)}{-h}=\widetilde{f}^{\prime}\left(x_{0}\right),
\end{aligned}
$$

or: (iii) For all sufficiently small $h>0, \exists \widetilde{f}\left(x_{0}+h\right) \ominus$ $\tilde{f}\left(x_{0}\right), \exists \tilde{f}\left(x_{0}-h\right) \ominus \tilde{f}\left(x_{0}\right)$ and the limits, we have the following relation:

$$
\begin{aligned}
\lim _{h \rightarrow 0} & \frac{\tilde{f}\left(x_{0}+h\right) \ominus \tilde{f}\left(x_{0}\right)}{h} \\
\quad & \lim _{h \rightarrow 0} \frac{\tilde{f}\left(x_{0}-h\right) \ominus \tilde{f}\left(x_{0}\right)}{-h}=\tilde{f}^{\prime}\left(x_{0}\right),
\end{aligned}
$$

or:

(iv) For all sufficiently small $h>0, \exists \tilde{f}\left(x_{0}\right) \ominus \widetilde{f}\left(x_{0}+\right.$ $h), \exists \widetilde{f}\left(x_{0}\right) \ominus \widetilde{f}\left(x_{0}-h\right)$ and the limits, we have the following relation:

$$
\begin{aligned}
\lim _{h \rightarrow 0} & \frac{\tilde{f}\left(x_{0}\right) \ominus \tilde{f}\left(x_{0}+h\right)}{-h} \\
\quad & \lim _{h \rightarrow 0} \frac{\tilde{f}\left(x_{0}\right) \ominus \tilde{f}\left(x_{0}-h\right)}{h}=\tilde{f}^{\prime}\left(x_{0}\right) .
\end{aligned}
$$

Definition 5. Let $\tilde{f}:(a, b) \rightarrow E$. We say that $\tilde{f}$ is (i)-differentiable on $(a, b)$ if $\tilde{f}$ is differentiable in the sense (i) of Definition (4). Similarly, we can derive differentiability of relations (ii), (iii), and (iv) [18].

Definition 6. Let $\tilde{f}: T \rightarrow E$ be Hukuhara differentiable and be denoted by $[f(t)]^{r}=\left[f_{-}^{r}, f_{+}^{r}\right]$. Then, the boundary functions $f_{-}^{r}$ and $f_{+}^{r}$ are differentiable and:

$$
\left.f^{\prime}(t)\right]^{r}=\left[\left(f_{-}^{r}\right)^{\prime}(t),\left(f_{+}^{r}\right)^{\prime}(t)\right], \quad t \in T, \quad r \in[0,1] .
$$

Definition 7. We say that $\tilde{f}$ satisfies the condition $\left(H_{1}\right)$ at $x \in(a, b)$ if $\widetilde{f}(x+h) \ominus \widetilde{f}(x)$ and $\widetilde{f}(x) \ominus \widetilde{f}(x-$ $h$ ) exist sufficiently small for $h$. Also, we say that $\tilde{f}$ satisfies the condition $\left(H_{2}\right)$ at $x \in(a, b)$ if $\widetilde{f}(x) \ominus \widetilde{f}(x+$ $h)$ and $\tilde{f}(x-h) \ominus \widetilde{f}(x)$ exist for $h$ sufficiently small.

\section{Model formulation}

In this section, we propose a discount profit maximization inventory model in the fuzzy environment. This paper considers a fuzzy inventory system with perishable multi products, where the fuzzy selling price of products by retailer and scheduling fuzzy replenishment order quantity are evaluated periodically at each time $t, t=0,1,2, H$, where $H$ is the planning horizon. Moreover, we assume that the replenishment of products is immediate without in-transit inventory of the product and no shortage happens. Hence, the decision in each time period includes scheduling fuzzy replenishment order quantity and its associated fuzzy selling price of products. Therefore, this problem is proper to obtain the optimal order of issuance for new replenishment $z_{i-1}, i=1,2, \ldots, n$, with the selling price 
of products being reorganized by the retailer and the order quantities identified simultaneously to maximize the discount profit over $[0, H]$. It should be noted that the new replenishment of products at $z_{i-1}$ is an arrangement for a selling period over $\left[z_{i-1}, z_{i}\right]$ [10].

The fuzzy multivariate demand function considered in this study, which is shown by $\widetilde{H}\left(x_{d}, t\right)$, is defined as follows:

$$
\widetilde{H}\left(x_{d}, t\right)=\widetilde{f}\left(x_{d}\right) \widetilde{g}(t),
$$

where $d$ is number of products type, $\tilde{f}\left(x_{d}\right)=\left(\widetilde{a_{d}} \ominus\right.$ $\left.\widetilde{b}_{d} x_{d}\right)$; both $D\left(\widetilde{a}_{d}, \widetilde{0}\right) \geq 0, D\left(\widetilde{b}_{d}, \widetilde{0}\right) \geq 0$ and $\widetilde{g}(t)=e^{\widetilde{\lambda}_{d} t}$, since $D\left(e^{\widetilde{\lambda}_{d} t}, \widetilde{0}\right) \geq 0$ is nonnegative for all $\widetilde{\lambda}_{d}$. The fuzzy multivariate demand function should satisfy the following assumptions:

(i) $\widetilde{H}\left(x_{d}, t\right)$ is decreasing in $x_{d}$;

(ii) $\lim _{x_{d} \rightarrow 0} D\left(\widetilde{H}\left(x_{d}, t\right), \widetilde{0}\right)<+\infty$ and $\lim _{x_{d} \rightarrow 0} D(\widetilde{H}$ $\left.\left(x_{d}, t\right), \widetilde{0}\right)=0$ for $t \geq 0$

(iii) $D\left(\widetilde{H}\left(x_{d}, t\right), \widetilde{0}\right) \geq 0$ for $x_{d}>0$ and $t \geq 0$.

It should be noted that the intended fuzzy multivariate demand function satisfies the three assumptions above. Moreover, $\widetilde{\lambda}_{d}$ is the sales fuzzy trend of each product over a life cycle. This parameter indicates that the demand rate changes over the planning horizon. On the other hand, since the objective is the maximization of the profit, the total cost should be deducted from the total revenue. Since these costs, including inventory costs, involve deterioration, replenishment, production, holding costs, etc., in general, all factors which are effective in the calculation of revenue and costs over time are subject to discount and inflation rates. During the planning period, the net discount rate of inflation is assumed constant; that is $R=\psi-\eta$, where $\eta$ is the inflation rate and $\psi$ is the discount rate, demonstrating the time value of money. The discounted cash flow value is calculated by $Y_{t} e^{-R t}$ for $t \geq 0$, where $Y_{t}$ is the value of $Y$ at time $t$. The following notations are utilized throughout this study:
$\widetilde{c}_{d}: \quad$ Per unit production cost of product type $d$ for the manufacturer;
$\widetilde{h}_{d} \quad$ Per unit holding cost of product type $d$ for a given unit of time;
$\widetilde{w}_{d}: \quad$ Per unit purchase cost of product type $d$ for the retailer;
$\tilde{\theta}_{d}: \quad$ Deterioration rate of product type $d$;
$\widetilde{s}_{x_{d}}: \quad$ Replenishment operations cost of product type $d$ per lot;

$\widetilde{s}_{M_{d}}$ : $\quad$ Production setup cost of product type $d$ per run;

$\widetilde{A}_{R_{d}}: \quad$ Per unit transaction cost of product type $d$ for the retailer;

$\widetilde{A}_{M_{d}}$ : $\quad$ Per unit transaction cost of product type $d$ for the manufacturer;

$\widetilde{u}_{t}\left(x_{d}, t\right)$ : Inventory of product type $d$ at time $t$;

$\widetilde{Q}\left(x_{d}\right)$ : $\quad$ Replenishment quantity of product type $d$;

$\tilde{\pi}\left(x_{d}, t\right)$ : $\quad$ Profit obtained for product type $d$ over period $t \in\left[z_{i-1}, z_{i}\right]$;

$\widetilde{\Pi}_{z_{i}}\left(x_{d}, t\right)$ : Profit obtained for product type $d$ over period $t \in\left[0, z_{i}\right]$.

Also, in order to get more realistic results, we assumed that the deterioration rate of products is variable and time-dependent.

Based on the aforementioned descriptions and with respect to the demand requirement and effect of deterioration rate over a selling period $\left[z_{i-1}, z_{i}\right]$, the inventory at time $t$ in the fuzzy environment can be denoted by the differential equation as follows:

$$
\begin{gathered}
\widetilde{u}_{t}\left(x_{d}, t\right)=\ominus \widetilde{\theta}_{d} \widetilde{u}\left(x_{d}, t\right) \ominus\left(\widetilde{a}_{d} \ominus \widetilde{b}_{d} x_{d}\right) e^{\tilde{\lambda}_{d} t} \\
0 \leq z_{k-1} \leq t \leq z_{k} \leq T
\end{gathered}
$$

where:

$$
\tilde{\theta}_{d}=f(t) \text {. }
$$

By utilizing Eq. (1), the replenished-order quantity is the inventory level at the start of replenishment and can be calculated as follows:

$$
\widetilde{Q}\left(x_{d}\right)=\widetilde{u}\left(x_{d}, z_{k-1}\right) .
$$

Based on the aforementioned considerations, we can calculate discount profit of the retailer in the selling period $\left[z_{i-1}, z_{i}\right]$ based on the total revenue and various costs as follows:

$$
\begin{aligned}
\widetilde{\pi}\left(x_{d}, t\right)_{R} & =\sum_{j=z_{k-1}}^{z_{k}} x_{d} e^{-R t_{j}}\left(\widetilde{a} \ominus \widetilde{b} x_{d}\right) e^{\lambda t_{j}} \\
& \ominus \widetilde{w}_{d} e^{-R z_{k}-1} \sum_{i=0}^{n} \widetilde{a}_{i} p_{i}^{\alpha, \beta}\left(x_{d}\right) p_{i}^{\alpha, \beta}\left(z_{k-1}\right) \\
& \ominus \sum_{j=z_{k-1}}^{z_{k}} \widetilde{h}_{d} e^{-R t_{j}} \sum_{i=0}^{n} \widetilde{a}_{i} p_{i}^{\alpha, \beta}\left(x_{d}\right) p_{i}^{\alpha, \beta}(t) \\
& \ominus \widetilde{A}_{R_{d}} e^{-R z_{k-1}} \sum_{i=0}^{n} \widetilde{a}_{i} p_{i}^{\alpha, \beta}\left(x_{d}\right) p_{i}^{\alpha, \beta}\left(z_{k-1}\right) \\
& \widetilde{S}_{R_{d}} e^{-R z_{k-1}} .
\end{aligned}
$$


Moreover, we can calculate discount profit of the manufacturer in the selling period $\left[z_{i-1}, z_{i}\right]$ based on the total revenue and various costs as follows:

$$
\begin{aligned}
\widetilde{\pi}\left(x_{d}, t\right)_{M} & =\widetilde{w}_{d} e^{-R z_{k-1}} \sum_{i=0}^{n} \widetilde{a}_{i} p_{i}^{\alpha, \beta}\left(x_{d}\right) p_{i}^{\alpha, \beta}\left(z_{k-1}\right) \\
& \ominus \widetilde{c} e^{-R z_{k-1}} \sum_{i=0}^{n} \widetilde{a}_{i} p_{i}^{\alpha, \beta}\left(x_{d}\right) p_{i}^{\alpha, \beta}\left(z_{k-1}\right) \\
& \ominus \widetilde{A}_{M_{d}} e^{-R z_{k-1}} \sum_{i=0}^{n} \widetilde{a}_{i} p_{i}^{\alpha, \beta}\left(x_{d}\right) p_{i}^{\alpha, \beta}\left(z_{k-1}\right) \\
& \ominus \widetilde{S}_{M_{d}} e^{-R z_{k-1}} .
\end{aligned}
$$

Since the proposed model is a fuzzy partial differential equation, to obtain a fuzzy decision, we have to use the advanced fuzzy methods, such as fuzzy collocation approaches, instead of regular fuzzy methods. This is because in these methods, parameters and data are fuzzy numbers, but the values obtained for the objective function and decision variables are deterministic.

\section{Solution methodology}

In this section we describe the proposed fuzzy expansion methods including Jacobi polynomials, airfoil polynomials and fuzzy collocation methods for solving proposed model.

\subsection{Description of the Jacobi polynomials method}

In this section, we describe the proposed fuzzy expansion methods including Jacobi polynomials, airfoil polynomials, and fuzzy collocation methods for solving the proposed model.

To obtain the approximation solution of Eq. (1), according to the Jacobli polynomials method [19], we can write:

$$
\begin{aligned}
& \widetilde{u}_{n}\left(x_{d}, t\right)=w\left(x_{d}\right) w(t) \sum_{i=0}^{n} \widetilde{a}_{i} p_{i}^{\alpha, \beta}\left(x_{d}\right) p_{i}^{\alpha, \beta}(t), \\
& \alpha, \beta>-1,
\end{aligned}
$$

where:

$$
\begin{gathered}
w\left(x_{d}\right)=\frac{\left(1-x_{d}\right)^{\alpha}}{\left(1+x_{d}\right)^{\beta}}, \quad w(t)=\frac{(1-t)^{\alpha}}{(1+t)^{\beta}}, \\
p_{i}^{\alpha, \beta}\left(x_{d}\right)=\frac{\left(1-x_{d}\right)^{-\alpha}\left(1+x_{d}\right)^{-\beta}}{(-2)^{i} i !} \\
\frac{d^{i}}{d x_{d}^{i}}\left[\left(1-x_{d}\right)^{i+\alpha}\left(1+x_{d}\right)^{i+\beta}\right], \\
\left(p_{i}^{\alpha, \beta}\right)^{\prime}\left(x_{d}\right)=\frac{1}{2}(i+\alpha+\beta+1) p_{i-1}^{(\alpha+1, \beta+1)}\left(x_{d}\right),
\end{gathered}
$$

$$
\begin{aligned}
& p_{i}^{\alpha, \beta}(t)=\frac{(1-t)^{-\alpha}(1+t)^{-\beta}}{(-2)^{i} n i !} \frac{d^{i}}{d t^{i}}\left[(1-t)^{i+\alpha}(1+t)^{i+\beta}\right] \\
& \left(p_{i}^{\alpha, \beta}\right)^{\prime}(t)=\frac{1}{2}(i+\alpha+\beta+1) p_{i-1}^{(\alpha+1, \beta+1)}(t)
\end{aligned}
$$

Now, from airfoil polynomials method, we have three cases as follows:

Case (1): If $\sum_{i=0}^{n} \widetilde{a}_{i} p_{i}^{\alpha, \beta}\left(x_{d}\right) p_{i}^{\alpha, \beta}(t)$ is $(i)$-differentiable, then $w\left(x_{d}\right) w(t) \sum_{i=0}^{n} \widetilde{a}_{i} p_{i}^{\alpha, \beta}\left(x_{d}\right) p_{i}^{\alpha, \beta}(t)$ is $(i)$ differentiable and we have:

$$
\begin{aligned}
& \frac{\partial}{\partial t} \widetilde{u}_{n}\left(x_{d}, t\right)=\widetilde{u}_{n}\left(x_{d}, t\right) \\
&=\left(w\left(x_{d}\right) w(t) \cdot \sum_{i=0}^{n} \widetilde{a}_{i} p_{i}^{\alpha, \beta}\left(x_{d}\right)\left(p_{i}^{\alpha, \beta}(t)\right)^{\prime}\right. \\
&=w\left(x_{d}\right) w^{\prime}(t) \sum_{i=0}^{n} \widetilde{a}_{i} p_{i}^{\alpha, \beta}\left(x_{d}\right) p_{i}^{\alpha, \beta}(t) \\
&\left.\oplus w_{d}\right) w(t) \sum_{i=0}^{n} \widetilde{a}_{i} p_{i}^{\alpha, \beta}\left(x_{d}\right)\left(p_{i}^{\alpha, \beta}\right)^{\prime}(t) \\
&=\sum_{i=0}^{n} \widetilde{a}_{i} w^{\prime}(t) w\left(x_{d}\right) p_{i}^{\alpha, \beta}\left(x_{d}\right) p_{i}^{\alpha, \beta}(t) \\
& \oplus \sum_{i=0}^{n} \widetilde{a}_{i} w\left(x_{d}\right) w(t)\left(p_{i}^{\alpha, \beta}\right)^{\prime}(t) p_{i}^{\alpha, \beta}\left(x_{d}\right) \\
& p_{i-1}^{(\alpha+1, \beta+1)}(t) p_{i}^{\alpha, \beta}\left(x_{d}\right) . \\
&=\sum_{i=0}^{n} \widetilde{a}_{i} w^{\prime}(t) w\left(x_{d}\right) p_{i}^{\alpha, \beta}\left(x_{d}\right) p_{i}^{\alpha, \beta}(t) \\
& \oplus \sum_{i=0}^{n} \widetilde{a}_{i} w\left(x_{d}\right) w(t) \frac{1}{2}(i+\alpha+\beta+1) \\
& \\
& \\
&
\end{aligned}
$$

Case (2): If $\sum_{i=0}^{n} \widetilde{a}_{i} p_{i}^{\alpha, \beta}\left(x_{d}\right) p_{i}^{\alpha, \beta}(t)$ is (ii)-differentiable and $w\left(x_{d}\right) w(t) \sum_{i=0}^{n} \widetilde{a}_{i} p_{i}^{\alpha, \beta}\left(x_{d}\right) p_{i}^{\alpha, \beta}(t)$ satisfies $\left(H_{1}\right)$ at $t$, then $w\left(x_{d}\right) w(t) \sum_{i=0}^{n} \widetilde{a}_{i} p_{i}^{\alpha, \beta}\left(x_{d}\right) p_{i}^{\alpha, \beta}(t)$ is (ii)-differentiable and we have:

$$
\begin{aligned}
\widetilde{u}_{n}^{\prime}\left(x_{d}, t\right) & =\left(w\left(x_{d}\right) w(t) \cdot \sum_{i=0}^{n} \widetilde{a}_{i}\left(p_{i}^{\alpha, \beta}(t)\right)^{\prime} p_{i}^{\alpha, \beta}\left(x_{d}\right)\right. \\
& =w^{\prime}(t) w\left(x_{d}\right) \sum_{i=0}^{n} \widetilde{a}_{i} p_{i}^{\alpha, \beta}(t) p_{i}^{\alpha, \beta}\left(x_{d}\right) \\
& \ominus\left(-w\left(x_{d}\right) w(t)\right) \sum_{i=0}^{n} \widetilde{a}_{i}\left(p_{i}^{\alpha, \beta}\right)^{\prime}(t) p_{i}^{\alpha, \beta}\left(x_{d}\right)
\end{aligned}
$$




$$
\begin{aligned}
& =\sum_{i=0}^{n} \widetilde{a}_{i} w^{\prime}(t) w\left(x_{d}\right) p_{i}^{\alpha, \beta}\left(x_{d}\right) p_{i}^{\alpha, \beta}(t) \\
& \ominus \sum_{i=0}^{n} \widetilde{a}_{i}\left(-w\left(x_{d}\right) w(t)\right)\left(p_{i}^{\alpha, \beta}\right)^{\prime}(t) p_{i}^{\alpha, \beta}\left(x_{d}\right) \\
& =\sum_{i=0}^{n} \widetilde{a}_{i} w^{\prime}(t) w\left(x_{d}\right) p_{i}^{\alpha, \beta}\left(x_{d}\right) p_{i}^{\alpha, \beta}(t) \\
& \ominus \sum_{i=0}^{n} \widetilde{a}_{i}\left(-w\left(x_{d}\right) w(t)\right) \frac{1}{2}(i+\alpha+\beta+1) \\
& p_{i-1}^{(\alpha+1, \beta+1)}(t) p_{i}^{\alpha, \beta}\left(x_{d}\right) .
\end{aligned}
$$

Case (3): If $\sum_{i=0}^{n} \widetilde{a}_{i} p_{i}^{\alpha, \beta}\left(x_{d}\right) p_{i}^{\alpha, \beta}(t)$ is (ii)-differentiable and $w\left(x_{d}\right) w(t) \sum_{i=0}^{n} \tilde{a}_{i} p_{i}^{\alpha, \beta}\left(x_{d}\right) p_{i}^{\alpha, \beta}(t)$ satisfies $\left(H_{2}\right)$ at $t$, then $w\left(x_{d}\right) w(t) \sum_{i=0}^{n} \widetilde{a}_{i} p_{i}^{\alpha, \beta}\left(x_{d}\right) p_{i}^{\alpha, \beta}(t)$ is (ii)-differentiable and we have:

$$
\begin{aligned}
& \widetilde{u}_{n}^{\prime}\left(x_{d}, t\right)=\left(w\left(x_{d}\right) w(t) \sum_{i=0}^{n} \widetilde{a}_{i} p_{i}^{\alpha, \beta}(t)\right)^{\prime} p_{i}^{\alpha, \beta}\left(x_{d}\right) \\
& =w\left(x_{d}\right) w(t) \sum_{i=0}^{n} \widetilde{a}_{i}\left(p_{i}^{\alpha, \beta}\right)^{\prime}(t) p_{i}^{\alpha, \beta}\left(x_{d}\right) \\
& \ominus\left(-w^{\prime}(t) w\left(x_{d}\right)\right) \sum_{i=0}^{n} \widetilde{a}_{i} p_{i}^{\alpha, \beta}\left(x_{d}\right) p_{i}^{\alpha, \beta}(t) \\
& =\sum_{i=0}^{n} \widetilde{a}_{i} w\left(x_{d}\right) w(t)\left(p_{i}^{\alpha, \beta}\right)^{\prime}(t) p_{i}^{\alpha, \beta}\left(x_{d}\right) \\
& \ominus \sum_{i=0}^{n} \widetilde{a}_{i}\left(-w^{\prime}(t) w\left(x_{d}\right) p_{i}^{\alpha, \beta}\left(x_{d}\right) p_{i}^{\alpha, \beta}(t)\right. \\
& =\sum_{i=0}^{n} \widetilde{a}_{i} w\left(x_{d}\right) w(t) \frac{1}{2}(i+\alpha+\beta+1) \\
& p_{i-1}^{(\alpha+1, \beta+1)}(t) p_{i}^{\alpha, \beta}\left(x_{d}\right) \\
& \ominus \sum_{i=0}^{n} \widetilde{a}_{i}\left(-w^{\prime}(t) w\left(x_{d}\right)\right) p_{i}^{\alpha, \beta}\left(x_{d}\right) p_{i}^{\alpha, \beta}(t) .
\end{aligned}
$$

Based on the aforementioned considerations, we can write Eq. (5) as follows:

$$
\begin{aligned}
& \ominus f(t) w\left(x_{d}\right) w(t) \sum_{i=0}^{n} \widetilde{a}_{i} p_{i}^{\alpha, \beta}\left(x_{d}\right) p_{i}^{\alpha, \beta}(t) \ominus\left(\widetilde{a}_{d} \ominus \widetilde{b}_{d} x_{d}\right) e^{\tilde{\lambda}_{d} t} \\
& =\left(w\left(x_{d}\right) w(t) \sum_{i=0}^{n} \widetilde{a}_{i} p_{i}^{\alpha, \beta}\left(x_{d}\right) p_{i}^{\alpha, \beta}(t)\right)^{\prime} \oplus \widetilde{R}_{n}\left(x_{d}, t\right)
\end{aligned}
$$

Thus, we have:

$$
\begin{aligned}
& \ominus f(t) w\left(x_{d}\right) w(t) \sum_{i=0}^{n} \widetilde{a}_{i} p_{i}^{\alpha, \beta}\left(x_{d}\right) p_{i}^{\alpha, \beta}(t) \\
& \ominus\left(\widetilde{a}_{d} \ominus \widetilde{b}_{d} x_{d}\right) e^{\widetilde{\lambda}_{d} t} \\
& \ominus\left(w\left(x_{d}\right) w(t) \sum_{i=0}^{n} \widetilde{a}_{i} p_{i}^{\alpha, \beta}\left(x_{d}\right) p_{i}^{\alpha, \beta}(t)\right)^{\prime} \\
& =\widetilde{R}_{n}\left(x_{d}, t\right), \\
& \widetilde{R}_{n}\left(x_{d j}, t_{j}\right)=\widetilde{0} .
\end{aligned}
$$

It means that:

$$
\underline{R}_{n}^{r}\left(x_{d j}, t_{j}\right)=0, \quad \bar{R}_{n}^{r}\left(x_{d j}, t_{j}\right)=0, \quad \forall r \in[0,1],
$$

where, $x_{d j}$ and $t_{j}(j=1, \cdots, n)$ are collocation points. Therefore, we can write:

$$
\begin{aligned}
& \ominus f\left(t_{j}\right) w\left(x_{d j}\right) w\left(t_{j}\right) \sum_{i=0}^{n} \widetilde{a}_{i} p_{i}^{\alpha, \beta}\left(x_{d j}\right) p_{i}^{\alpha, \beta}\left(t_{j}\right) \\
& \ominus\left(\widetilde{a}_{d} \ominus \widetilde{b}_{d} x_{d j}\right) e^{\tilde{\lambda}_{d} t_{j}} \\
& \ominus\left(w\left(x_{d j}, t_{j}\right) \sum_{i=0}^{n} \widetilde{a}_{i} p_{i}^{\alpha, \beta}\left(x_{d j}\right) p_{i}^{\alpha, \beta}\left(t_{j}\right)\right)^{\prime}=\widetilde{0}
\end{aligned}
$$

Depending on the type of derivative that was defined in Eq. (7), for $\left(w\left(x_{d}\right) w(t) \sum_{i=0}^{n} \widetilde{a}_{i} p_{i}^{\alpha, \beta}\left(x_{d}\right) p_{i}^{\alpha, \beta}(t)\right)^{\prime}$ Eq. (5) can be written as follows when $\sum_{i=0}^{n} \widetilde{a}_{i} p_{i}^{\alpha, \beta}$ $\left(x_{d j}\right) p_{i}^{\alpha, \beta}\left(t_{j}\right)$ is $(i)$-differentiable:

$$
\begin{gathered}
\ominus f\left(t_{j}\right) w\left(x_{d j}\right) w\left(t_{j}\right) \sum_{i=0}^{n} \widetilde{a}_{i} p_{i}^{\alpha, \beta}\left(x_{d j}\right) p_{i}^{\alpha, \beta}\left(t_{j}\right) \\
\ominus\left(\widetilde{a}_{d} \ominus \widetilde{b}_{d} x_{d j}\right) e^{\tilde{\lambda}_{d} t_{j}} \\
\ominus\left(\sum_{i=0}^{n} \widetilde{a}_{i} w^{\prime}\left(t_{j}\right) w\left(x_{d j}\right) p_{i}^{\alpha, \beta}\left(x_{d j}\right) p_{i}^{\alpha, \beta}\left(t_{j}\right)\right. \\
\oplus \sum_{i=0}^{n} \widetilde{a}_{i} w\left(x_{d j}\right) w\left(t_{j}\right) \frac{1}{2}(n+\alpha+\beta+1) \\
\left.p_{n-1}^{(\alpha+1, \beta+1)}(t) p_{i}^{\alpha, \beta}\left(x_{d j}\right)\right)=\widetilde{0} .
\end{gathered}
$$

Eq. (14) can be written in the following operator form:

$$
\ominus A \widetilde{a} \ominus \widetilde{H} \ominus(B \widetilde{a} \oplus C \widetilde{a})=\widetilde{0},
$$




$$
\begin{aligned}
\sum_{a_{i j} \geq 0} a_{i j} \widetilde{a}_{j} \oplus \sum_{f_{i j}<0} f_{i j} \widetilde{a}_{j} \ominus \widetilde{h}_{i j} \ominus\left(\sum_{b_{i j} \geq 0} b_{i j} \widetilde{a}_{j}\right. \\
\left.\oplus \sum_{b_{i j}<0} b_{i j} \widetilde{a}_{j} \oplus \sum_{c_{i j} \geq 0} c_{i j} \widetilde{a}_{j} \oplus \sum_{c_{i j}<0} c_{i j} \widetilde{a}_{j}\right)=\widetilde{0}
\end{aligned}
$$

where:

$$
\begin{aligned}
(A)_{i j}= & f\left(t_{j}\right) w\left(x_{d j}\right) w\left(t_{j}\right) p_{i}^{\alpha, \beta}\left(x_{d j}\right) p_{i}^{\alpha, \beta}\left(t_{j}\right), \\
(\widetilde{H})_{i j}= & \left(\widetilde{a}_{d} \ominus \widetilde{b}_{d} x_{d j}\right) e^{\tilde{\lambda}_{d} t_{j}} \\
(B)_{i j}= & w^{\prime}\left(t_{j}\right) w\left(x_{d j}\right) p_{i}^{\alpha, \beta}\left(x_{d j}\right) p_{i}^{\alpha, \beta}\left(t_{j}\right), \\
(C)_{i j}= & w\left(x_{d j}\right) w\left(t_{j}\right) \frac{1}{2}(n+\alpha+\beta+1) \\
& p_{n-1}^{(\alpha+1, \beta+1)}(t) p_{i}^{\alpha, \beta}\left(x_{d j}\right) .
\end{aligned}
$$

Depending on the type of derivative that was defined in Eq. (8), for $\left(w\left(x_{d}\right) w(t) \sum_{i=0}^{n} \widetilde{a}_{i} p_{i}^{\alpha, \beta}\left(x_{d}\right) p_{i}^{\alpha, \beta}(t)\right)^{\prime}$, Eq. (5) can be written as follows when $\sum_{i=0}^{n}$ $\tilde{a}_{i} p_{i}^{\alpha, \beta}\left(x_{d j}\right) p_{i}^{\alpha, \beta}\left(t_{j}\right)$ is $(i i)$-differentiable and $w\left(x_{d}\right) w(t)$ $\sum_{i=0}^{n} \widetilde{a}_{i} p_{i}^{\alpha, \beta}\left(x_{d}\right) p_{i}^{\alpha, \beta}(t)$ satisfies condition $H_{1}$ :

$$
\begin{aligned}
& \ominus f\left(t_{j}\right) w\left(x_{d j}\right) w\left(t_{j}\right) \sum_{i=0}^{n} \widetilde{a}_{i} p_{i}^{\alpha, \beta}\left(x_{d j}\right) p_{i}^{\alpha, \beta}\left(t_{j}\right) \\
& \ominus\left(\widetilde{a}_{d} \ominus \widetilde{b}_{d} x_{d j}\right) e^{\widetilde{\lambda}_{d} t_{j}} \\
& \ominus\left(\sum_{i=0}^{n} \widetilde{a}_{i} w^{\prime}\left(t_{j}\right) w\left(x_{d j}\right) p_{i}^{\alpha, \beta}\left(x_{d j}\right) p_{i}^{\alpha, \beta}\left(t_{j}\right)\right. \\
& \ominus \sum_{i=0}^{n} \widetilde{a}_{i}\left(-w\left(x_{d j}\right) w\left(t_{j}\right)\right) \frac{1}{2}(n+\alpha+\beta+1) \\
& \left.p_{n-1}^{(\alpha+1, \beta+1)}\left(t_{j}\right) p_{i}^{\alpha, \beta}\left(x_{d j}\right)\right)=\widetilde{0} .
\end{aligned}
$$

Eq. (17) can be written in the following operator form:

$$
\begin{aligned}
& \ominus A \widetilde{a} \ominus \widetilde{H} \ominus(B \widetilde{a} \ominus C \widetilde{a})=\widetilde{0} \\
& \sum_{a_{i j} \geq 0} a_{i j} \widetilde{a}_{j} \oplus \sum_{f_{i j}<0} f_{i j} \widetilde{a}_{j} \ominus \widetilde{h}_{i j} \ominus\left(\sum_{b_{i j} \geq 0} b_{i j} \widetilde{a}_{j}\right. \\
& \left.\oplus \sum_{b_{i j}<0} b_{i j} \widetilde{a}_{j} \ominus \sum_{c_{i j} \geq 0} c_{i j} \widetilde{a}_{j} \oplus \sum_{c_{i j}<0} c_{i j} \widetilde{a}_{j}\right)=\widetilde{0}
\end{aligned}
$$

where:

$$
\begin{aligned}
(A)_{i j} & =f\left(t_{j}\right) w\left(x_{d j}\right) w\left(t_{j}\right) p_{i}^{\alpha, \beta}\left(x_{d j}\right) p_{i}^{\alpha, \beta}\left(t_{j}\right), \\
(\widetilde{H})_{i j} & =\left(\widetilde{a}_{d} \ominus \widetilde{b}_{d} x_{d j}\right) e^{\tilde{\lambda}_{d} t_{j}} \\
(B)_{i j} & =w^{\prime}\left(t_{j}\right) w\left(x_{d j}\right) p_{i}^{\alpha, \beta}\left(x_{d j}\right) p_{i}^{\alpha, \beta}\left(t_{j}\right), \\
(C)_{i j}= & \left(-w\left(x_{d j}\right) w\left(t_{j}\right)\right) \frac{1}{2}(n+\alpha+\beta+1) \\
& p_{n-1}^{(\alpha+1, \beta+1)}\left(t_{j}\right) p_{i}^{\alpha, \beta}\left(x_{d j}\right) .
\end{aligned}
$$

Depending on the type of derivative that was defined in Eq. (9), for $\left.\left(w\left(x_{d}\right) w(t) \sum_{i=0}^{n} \widetilde{a}_{i} p_{i}^{\alpha, \beta}\left(x_{d}\right) p^{\alpha, \beta}(t)\right)\right)^{\prime}$ Eq. (5) can be written as follows when $\sum_{i=0}^{n}$ $\widetilde{a}_{i} p_{i}^{\alpha, \beta}\left(x_{d j}\right) p_{i}^{\alpha, \beta}\left(t_{j}\right)$ is $(i i)$-differentiable and $w\left(x_{d}\right) w(t)$ $\sum_{i=0}^{n} \widetilde{a}_{i} p_{i}^{\alpha, \beta}\left(x_{d}\right) p_{i}^{\alpha, \beta}(t)$ satisfies condition $\mathrm{H}_{2}$ :

$$
\begin{aligned}
& \ominus f\left(t_{j}\right) w\left(x_{d j}\right) w\left(t_{j}\right) \sum_{i=0}^{n} \widetilde{a}_{i} p_{i}^{\alpha, \beta}\left(x_{d j}\right) p_{i}^{\alpha, \beta}\left(t_{j}\right) \\
& \ominus\left(\widetilde{a}_{d} \ominus \widetilde{b}_{d} x_{d j}\right) e^{\tilde{\lambda}_{d} t_{j}} \\
& \ominus\left(\sum_{i=0}^{n} \widetilde{a}_{i} w\left(x_{d j}\right) w\left(t_{j}\right) \frac{1}{2}(n+\alpha+\beta+1)\right. \\
& p_{n-1}^{(\alpha+1, \beta+1)}\left(t_{j}\right) p_{i}^{\alpha, \beta}\left(x_{d j}\right) \\
& \left.\ominus \sum_{i=0}^{n} \widetilde{a}_{i}\left(-w^{\prime}\left(t_{j}\right) w\left(x_{d j}\right)\right) p_{i}^{\alpha, \beta}\left(x_{d j}\right) p_{i}^{\alpha, \beta}\left(t_{j}\right)\right)=\widetilde{0} .
\end{aligned}
$$

Eq. (20) can be written in the following operator form:

$$
\begin{aligned}
& \ominus A \widetilde{a} \ominus \widetilde{H} \ominus(B \widetilde{a} \ominus C \widetilde{a})=\widetilde{0} \\
& \sum_{a_{i j} \geq 0} a_{i j} \widetilde{a}_{j} \oplus \sum_{f_{i j}<0} f_{i j} \widetilde{a}_{j} \ominus \widetilde{h}_{i j} \ominus\left(\sum_{b_{i j} \geq 0} b_{i j} \widetilde{a}_{j}\right. \\
& \left.\oplus \sum_{b_{i j}<0} b_{i j} \tilde{a}_{j} \ominus \sum_{c_{i j} \geq 0} c_{i j} \tilde{a}_{j} \oplus \sum_{c_{i j}<0} c_{i j} \tilde{a}_{j}\right)=\widetilde{0}
\end{aligned}
$$

where:

$$
\begin{aligned}
(A)_{i j}= & f\left(t_{j}\right) w\left(x_{d j}\right) w\left(t_{j}\right) p_{i}^{\alpha, \beta}\left(x_{d j}\right) p_{i}^{\alpha, \beta}\left(t_{j}\right), \\
(\widetilde{H})_{i j}= & \left(\widetilde{a}_{d} \ominus \widetilde{b}_{d} x_{d j}\right) e^{\tilde{\lambda}_{d} t_{j}} \\
(B)_{i j}= & w\left(x_{d j}\right) w\left(t_{j}\right) \frac{1}{2}(n+\alpha+\beta+1) \\
& p_{n-1}^{(\alpha+1, \beta+1)}\left(t_{j}\right) p_{i}^{\alpha, \beta}\left(x_{d j}\right)
\end{aligned}
$$$$
(C)_{i j}=\left(-w^{\prime}\left(t_{j}\right) w\left(x_{d j}\right)\right) p_{i}^{\alpha, \beta}\left(x_{d j}\right) p_{i}^{\alpha, \beta}\left(t_{j}\right) .
$$ 


\subsection{Description of the Airfoil polynomials method}

In order to obtain the approximation solution of Eq. (1), according to the airfoil polynomials fuzzy collocation method [20], we can write this equation as follows:

$$
\widetilde{u}_{n}\left(x_{d}, t\right)=w\left(x_{d}\right) w(t) \sum_{i=0}^{n} \widetilde{a}_{i} s_{i}\left(x_{d}\right) s_{i}(t)
$$

where:

$$
\begin{aligned}
& w\left(x_{d}\right)=\sqrt{\frac{1+x_{d}}{1-x_{d}}}, \\
& w(t)=\sqrt{\frac{1+t}{1-t}}, \\
& s_{i}\left(x_{d}\right)=\frac{\cos \left[\left(i+\frac{1}{2}\right) \arccos x_{d}\right]}{\cos \left(\frac{1}{2} \arccos x_{d}\right)}, \\
& s_{i}(t)=\frac{\cos \left[\left(i+\frac{1}{2}\right) \arccos t\right]}{\cos \left(\frac{1}{2} \arccos t\right)}, \\
& u_{i}\left(x_{d}\right)=\frac{\sin \left[\left(i+\frac{1}{2}\right) \arcsin x_{d}\right]}{\cos \left(\frac{1}{2} \arcsin x_{d}\right)}, \\
& u_{i}(t)=\frac{\sin \left[\left(i+\frac{1}{2}\right) \arcsin t\right]}{\cos \left(\frac{1}{2} \arcsin t\right)}, \\
& \left(1+x_{d}\right) s_{i}^{\prime}\left(x_{d}\right)=\left(i+\frac{1}{2}\right) u_{i}\left(x_{d}\right)-\frac{1}{2} s_{i}\left(x_{d}\right), \\
& (1+t) s_{i}^{\prime}(t)=\left(i+\frac{1}{2}\right) u_{i}(t)-\frac{1}{2} s_{i}(t) .
\end{aligned}
$$

Now, from airfoil polynomials method, we have three cases as follows:

Case (1): If $\sum_{i=0}^{n} \widetilde{a}_{i} s_{i}\left(x_{d}\right) s_{i}(t)$ is (i)-differentiable, then $w\left(x_{d}\right) w(t) \sum_{i=0}^{n} \widetilde{a}_{i} s_{i}\left(x_{d}\right) s_{i}(t)$ is $(i)$-differentiable and we have:

$$
\begin{aligned}
& \frac{\partial}{\partial t} \widetilde{u}_{n}\left(x_{d}, t\right)=\widetilde{u}_{n}^{\prime}\left(x_{d}, t\right) \\
& =\left(w\left(x_{d}\right) w(t) \cdot \sum_{i=0}^{n} \widetilde{a}_{i} s_{i}\left(x_{d}\right) s_{i}(t)\right)^{\prime} \\
& =w\left(x_{d}\right) w^{\prime}(t) \sum_{i=0}^{n} \widetilde{a}_{i} s_{i}\left(x_{d}\right) s_{i}(t) \\
& \oplus w\left(x_{d}\right) w(t) \sum_{i=0}^{n} \widetilde{a}_{i} s_{i}\left(x_{d}\right) s_{i}^{\prime}(t) \\
& =\sum_{i=0}^{n} \widetilde{a}_{i} w^{\prime}(t) w\left(x_{d}\right) s_{i}\left(x_{d}\right) s_{i}(t)
\end{aligned}
$$

$$
\begin{aligned}
& \oplus \sum_{i=0}^{n} \widetilde{a}_{i} w\left(x_{d}\right) w(t)\left(s_{i}\left(x_{d}\right) s_{i}^{\prime}(t)\right) \\
& =\sum_{i=0}^{n} \widetilde{a}_{i} w^{\prime}(t) w\left(x_{d}\right) s_{i}\left(x_{d}\right) s_{i}(t) \\
& \oplus \sum_{i=0}^{n} \widetilde{a}_{i} w\left(x_{d}\right) w(t)\left(\left(i+\frac{1}{2}\right) u_{i}(t)\right. \\
& \left.-\frac{1}{2} s_{i}(t)\right) s_{i}\left(x_{d}\right) .
\end{aligned}
$$

Case (2): If $\sum_{i=0}^{n} \widetilde{a}_{i} s_{i}\left(x_{d}\right) s_{i}(t)$ is (ii)-differentiable and $w\left(x_{d}\right) w(t) \sum_{i=0}^{n} \widetilde{a}_{i} s_{i}\left(x_{d}\right) s_{i}(t)$ satisfies $\left(H_{1}\right)$ at $t$, then $w\left(x_{d}\right) w(t) \sum_{i=0}^{n} \widetilde{a}_{i} s_{i}\left(x_{d}\right) s_{i}(t)$ is (ii)-differentiable and we have:

$$
\begin{aligned}
\widetilde{u}_{n}^{\prime}\left(x_{d}, t\right) & =\left(w\left(x_{d}\right) w(t) \cdot \sum_{i=0}^{n} \widetilde{a}_{i} s_{i}\left(x_{d}\right) s_{i}^{\prime}(t)\right. \\
& =w^{\prime}(t) w\left(x_{d}\right) \sum_{i=0}^{n} \widetilde{a}_{i} s_{i}\left(x_{d}\right) s_{i}(t) \\
& \ominus\left(-w\left(x_{d}\right) w(t)\right) \sum_{i=0}^{n} \widetilde{a}_{i} s_{i}\left(x_{d}\right) s_{i}^{\prime}(t) \\
& =\sum_{i=0}^{n} \widetilde{a}_{i} w^{\prime}(t) w\left(x_{d}\right) s_{i}\left(x_{d}\right) s_{i}(t) \\
& \ominus \sum_{i=0}^{n} \widetilde{a}_{i}\left(-w\left(x_{d}\right) w(t)\right) s_{i}\left(x_{d}\right) s_{i}^{\prime}(t) \\
& =\sum_{i=0}^{n} \widetilde{a}_{i} w^{\prime}(t) w\left(x_{d}\right) s_{i}\left(x_{d}\right) s_{i}(t) \\
& \ominus \sum_{i=0}^{n} \widetilde{a}_{i}\left(-w\left(x_{d}\right) w(t)\right)\left(\left(i+\frac{1}{2}\right) u_{i}(t)\right. \\
& \left.-\frac{1}{2} s_{i}(t)\right) s_{i}\left(x_{d}\right) .
\end{aligned}
$$

Case (3): If $\sum_{i=0}^{n} \widetilde{a}_{i} s_{i}\left(x_{d}\right) s_{i}(t)$ is (ii)-differentiable and $w\left(x_{d}\right) w(t) \sum_{i=0}^{n} \widetilde{a}_{i} s_{i}\left(x_{d}\right) s_{i}(t)$ satisfies $\left(H_{2}\right)$ at $t$, then $w\left(x_{d}\right) w(t) \sum_{i=0}^{n} \widetilde{a}_{i} s_{i}\left(x_{d}\right) s_{i}(t)$ is (ii)-differentiable and we have:

$$
\begin{aligned}
\widetilde{u}_{n}^{\prime}\left(x_{d}, t\right) & =\left(w\left(x_{d}\right) w(t) \sum_{i=0}^{n} \widetilde{a}_{i} s_{i}\left(x_{d}\right) s_{i}^{\prime}(t)\right. \\
& =w\left(x_{d}\right) w(t) \sum_{i=0}^{n} \widetilde{a}_{i} s_{i}\left(x_{d}\right) s_{i}^{\prime}(t)
\end{aligned}
$$




$$
\begin{aligned}
& \ominus\left(-w^{\prime}(t) w\left(x_{d}\right)\right) \sum_{i=0}^{n} \widetilde{a}_{i} s_{i}\left(x_{d}\right) s_{i}(t) \\
& =\sum_{i=0}^{n} \widetilde{a}_{i} w\left(x_{d}\right) w(t) s_{i}\left(x_{d}\right) s_{i}^{\prime}(t) \\
& \ominus \sum_{i=0}^{n} \widetilde{a}_{i}\left(-w^{\prime}(t) w\left(x_{d}\right) s_{i}\left(x_{d}\right) s_{i}(t)\right. \\
& =\sum_{i=0}^{n} \widetilde{a}_{i} w\left(x_{d}\right) w(t)\left(\left(i+\frac{1}{2}\right) u_{i}(t)-\frac{1}{2} s_{i}(t)\right) s_{i}\left(x_{d}\right) \\
& \ominus \sum_{i=0}^{n} \widetilde{a}_{i}\left(-w^{\prime}(t) w\left(x_{d}\right)\right) s_{i}\left(x_{d}\right) s_{i}(t) .
\end{aligned}
$$

We can write Eq. (5) as follows:

$$
\begin{gathered}
\ominus f(t) w\left(x_{d}\right) w(t) \sum_{i=0}^{n} \widetilde{a}_{i} s_{i}\left(x_{d}\right) s_{i}(t) \ominus\left(\widetilde{a}_{d} \ominus \widetilde{b}_{d} x_{d}\right) e^{\widetilde{\lambda}_{d} t} \\
=\left(w\left(x_{d}\right) w(t) \sum_{i=0}^{n} \widetilde{a}_{i} s_{i}\left(x_{d}\right) s_{i}(t)\right)^{\prime} \oplus \widetilde{R}_{n}\left(x_{d}, t\right)
\end{gathered}
$$

Thus, we have:

$$
\begin{gathered}
\ominus f(t) w\left(x_{d}\right) w(t) \sum_{i=0}^{n} \widetilde{a}_{i} s_{i}\left(x_{d}\right) s_{i}(t) \ominus\left(\widetilde{a}_{d} \ominus \widetilde{b}_{d} x_{d}\right) e^{\widetilde{\lambda}_{d} t} \\
\ominus\left(w\left(x_{d}\right) w(t) \sum_{i=0}^{n} \widetilde{a}_{i} s_{i}\left(x_{d}\right) s_{i}(t)\right)^{\prime}=\widetilde{R}_{n}\left(x_{d}, t\right) .
\end{gathered}
$$

By collocation method:

$$
\widetilde{R}_{n}\left(x_{d j}, t_{j}\right)=\widetilde{0} .
$$

\section{It means:}

$$
\underline{R}_{n}^{r}\left(x_{d j}, t_{j}\right)=0, \quad \bar{R}_{n}^{r}\left(x_{d j}, t_{j}\right)=0, \quad \forall r \in[0,1] .
$$

where, $x_{d j}$ and $t_{j}(j=1, \cdots, n)$ are collocation points.

$$
\begin{aligned}
& x_{d j}=-\cos \frac{2 j-1}{2 n+3} \pi, \quad j=0,1, \cdots, n, \\
& t_{j}=-\cos \frac{2 j-1}{2 n+3} \pi, \quad j=0,1, \cdots, n .
\end{aligned}
$$

Therefore, we can write:

$$
\begin{aligned}
\ominus f\left(t_{j}\right) w\left(x_{d j}\right) w\left(t_{j}\right) \sum_{i=0}^{n} \widetilde{a}_{i} s_{i}\left(x_{d j}\right) s_{i}\left(t_{j}\right) \\
\ominus\left(\widetilde{a}_{d} \ominus \widetilde{b}_{d} x_{d j}\right) e^{\tilde{\lambda}_{d} t_{j}} \\
\ominus\left(w\left(x_{d j}, t_{j}\right) \sum_{i=0}^{n} \widetilde{a}_{i} s_{i}\left(x_{d j}\right) s_{i}\left(t_{j}\right)\right)^{\prime}=\widetilde{0} .
\end{aligned}
$$

Depending on the type of derivative that was defined in Eq. (23), for $\left(w\left(x_{d}\right) w(t) \sum_{i=0}^{n} \widetilde{a}_{i} s_{i}\left(x_{d}\right) s_{i}(t)\right)^{\prime}$, Eq. (5) can be written as follows when $\sum_{i=0}^{n} \widetilde{a}_{i} s_{i}\left(x_{d j}\right) s_{i}\left(t_{j}\right)$ is (i)-differentiable:

$$
\begin{aligned}
\ominus f\left(t_{j}\right) & w\left(x_{d j}\right) w\left(t_{j}\right) \sum_{i=0}^{n} \widetilde{a}_{i} s_{i}\left(x_{d j}\right) s_{i}\left(t_{j}\right) \\
\ominus & \left(\widetilde{a}_{d} \ominus \widetilde{b}_{d} x_{d j}\right) e^{\tilde{\lambda}_{d} t_{j}} \\
\ominus & \left(\sum_{i=0}^{n} \widetilde{a}_{i} w^{\prime}\left(t_{j}\right) w\left(x_{d j}\right) s_{i}\left(x_{d j}\right) s_{i}\left(t_{j}\right)\right) \\
& \oplus \sum_{i=0}^{n} \widetilde{a}_{i} w\left(x_{d j}\right) w\left(t_{j}\right)\left(\left(i+\frac{1}{2}\right) u_{i}\left(t_{j}\right)\right. \\
- & \left.\frac{1}{2} s_{i}\left(t_{j}\right)\right) s_{i}\left(x_{d j}\right)=\widetilde{0} .
\end{aligned}
$$

Eq. (30) can be written in the following operator form:

$$
\begin{aligned}
& \ominus A \widetilde{a} \ominus \widetilde{H} \ominus(B \widetilde{a} \oplus C \widetilde{a})=\widetilde{0} \\
& \sum_{a_{i j} \geq 0} a_{i j} \widetilde{a}_{j} \oplus \sum_{f_{i j}<0} f_{i j} \widetilde{a}_{j} \ominus \widetilde{h}_{i j} \ominus\left(\sum_{b_{i j} \geq 0} b_{i j} \widetilde{a}_{j}\right. \\
& \left.\oplus \sum_{b_{i j}<0} b_{i j} \widetilde{a}_{j} \oplus \sum_{c_{i j} \geq 0} c_{i j} \widetilde{a}_{j} \oplus \sum_{c_{i j}<0} c_{i j} \widetilde{a}_{j}\right)=\widetilde{0}
\end{aligned}
$$

where:

$$
\begin{aligned}
& (A)_{i j}=f\left(t_{j}\right) w\left(x_{d j}\right) w\left(t_{j}\right) s_{i}\left(x_{d j}\right) s_{i}\left(t_{j}\right), \\
& (\widetilde{H})_{i j}=\left(\widetilde{a}_{d} \ominus \widetilde{b}_{d} x_{d j}\right) e^{\widetilde{\lambda}_{d} t_{j}},
\end{aligned}
$$$$
(B)_{i j}=w^{\prime}\left(t_{j}\right) w\left(x_{d j}\right) s_{i}\left(x_{d j}\right) s_{i}\left(t_{j}\right),
$$$$
(C)_{i j}=w\left(x_{d j}\right) w\left(t_{j}\right)\left(\left(i+\frac{1}{2}\right) u_{i}\left(t_{j}\right)-\frac{1}{2} s_{i}\left(t_{j}\right)\right) s_{i}\left(x_{d j}\right)
$$

Depending on the type of derivative that was defined in Eq. (24), for $\left(w\left(x_{d}\right) w(t) \sum_{i=0}^{n} \widetilde{a}_{i} s_{i}\left(x_{d}\right) s_{i}(t)\right)^{\prime}$, Eq. (5) can be written as follows when $\sum_{i=0}^{n} \widetilde{a}_{i} s_{i}\left(x_{d j}\right) s_{i}\left(t_{j}\right)$ is (ii)-differentiable and $w\left(x_{d j}\right) w\left(t_{j}\right) \sum_{i=0}^{n} \widetilde{a}_{i} s_{i}\left(x_{d j}\right) s_{i}\left(t_{j}\right)$ satisfies condition $H_{1}$ :

$$
\begin{gathered}
\ominus f\left(t_{j}\right) w\left(x_{d j}\right) w\left(t_{j}\right) \sum_{i=0}^{n} \widetilde{a}_{i} s_{i}\left(x_{d j}\right) s_{i}\left(t_{j}\right) \\
\ominus\left(\widetilde{a}_{d} \ominus \widetilde{b}_{d} x_{d j}\right) e^{\tilde{\lambda}_{d} t_{j}}
\end{gathered}
$$




$$
\begin{aligned}
& \ominus\left(\sum_{i=0}^{n} \widetilde{a}_{i} w^{\prime}\left(t_{j}\right) w\left(x_{d j}\right) s_{i}\left(x_{d j}\right) s_{i}\left(t_{j}\right)\right. \\
& \ominus \sum_{i=0}^{n} \widetilde{a}_{i}\left(-w\left(x_{d j}\right) w\left(t_{j}\right)\right)\left(\left(i+\frac{1}{2}\right) u_{i}\left(t_{j}\right)\right. \\
& \left.-\frac{1}{2} s_{i}\left(t_{j}\right)\right) s_{i}\left(x_{d j}\right)=\widetilde{0}
\end{aligned}
$$

Eq. (33) can be written in the following operator form:

$$
\begin{aligned}
& \ominus A \widetilde{a} \ominus \widetilde{H} \ominus(B \widetilde{a} \ominus C \widetilde{a})=\widetilde{0} \\
& \sum_{a_{i j} \geq 0} a_{i j} \widetilde{a}_{j} \oplus \sum_{f_{i j}<0} f_{i j} \widetilde{a}_{j} \ominus \widetilde{h}_{i j} \ominus\left(\sum_{b_{i j} \geq 0} b_{i j} \widetilde{a}_{j}\right. \\
& \left.\oplus \sum_{b_{i j}<0} b_{i j} \widetilde{a}_{j} \ominus \sum_{c_{i j} \geq 0} c_{i j} \widetilde{a}_{j} \oplus \sum_{c_{i j}<0} c_{i j} \widetilde{a}_{j}\right)=\widetilde{0},
\end{aligned}
$$

where:

$$
\begin{aligned}
(A)_{i j} & =f\left(t_{j}\right) w\left(x_{d j}\right) w\left(t_{j}\right) s_{i}\left(x_{d j}\right) s_{i}\left(t_{j}\right), \\
(\widetilde{H})_{i j} & =\left(\widetilde{a}_{d} \ominus \widetilde{b}_{d} x_{d j}\right) e^{\widetilde{\lambda}_{d} t_{j}}, \\
(B)_{i j} & =w^{\prime}\left(t_{j}\right) w\left(x_{d j}\right) s_{i}\left(x_{d j}\right) s_{i}\left(t_{j}\right), \\
(C)_{i j}= & \left(-w\left(x_{d j}\right) w\left(t_{j}\right)\right)\left(\left(i+\frac{1}{2}\right) u_{i}\left(t_{j}\right)\right. \\
& \left.-\frac{1}{2} s_{i}\left(t_{j}\right)\right) s_{i}\left(x_{d j}\right) .
\end{aligned}
$$

Depending on the type of derivative that was defined in Eq. (25), for $\left(w\left(x_{d}\right) w(t) \sum_{i=0}^{n} \widetilde{a}_{i} s_{i}\left(x_{d}\right) s_{i}(t)\right)^{\prime}$, Eq. (5) can be written as follows when $\sum_{i=0}^{n} \widetilde{a}_{i} s_{i}\left(x_{d j}\right) s_{i}\left(t_{j}\right)$ is (ii)-differentiable and $w\left(x_{d j}\right) w\left(t_{j}\right) \sum_{i=0}^{n} \widetilde{a}_{i} s_{i}\left(x_{d j}\right) s_{i}\left(t_{j}\right)$ satisfies condition $\mathrm{H}_{2}$ :

$$
\begin{aligned}
\ominus f\left(t_{j}\right) w\left(x_{d j}\right) w\left(t_{j}\right) \sum_{i=0}^{n} \widetilde{a}_{i} s_{i}\left(x_{d j}\right) s_{i}\left(t_{j}\right) \\
\ominus\left(\widetilde{a}_{d} \ominus \widetilde{b}_{d} x_{d j}\right) \tilde{\widetilde{\lambda}}^{\tilde{\lambda}_{j} t_{j}} \\
\ominus\left(\sum _ { i = 0 } ^ { n } \widetilde { a } _ { i } w ( x _ { d j } ) w ( t _ { j } ) \left(\left(i+\frac{1}{2}\right) u_{i}\left(t_{j}\right)\right.\right. \\
\left.\left.-\frac{1}{2} s_{i}\left(t_{j}\right)\right) s_{i}\left(x_{d j}\right)\right) \\
\ominus \sum_{i=0}^{n} \widetilde{a}_{i}\left(-w^{\prime}\left(t_{j}\right) w\left(x_{d j}\right)\right) s_{i}\left(x_{d j}\right) s_{i}\left(t_{j}\right)=\widetilde{0} .
\end{aligned}
$$

Eq. (36) can be written in the following operator form:

$$
\begin{aligned}
& \ominus A \widetilde{a} \ominus \widetilde{H} \ominus(B \widetilde{a} \ominus C \widetilde{a})=\widetilde{0} . \\
& \sum_{a_{i j} \geq 0} a_{i j} \widetilde{a}_{j} \oplus \sum_{f_{i j}<0} f_{i j} \widetilde{a}_{j} \ominus \widetilde{h}_{i j} \ominus\left(\sum_{b_{i j} \geq 0} b_{i j} \widetilde{a}_{j}\right. \\
& \left.\oplus \sum_{b_{i j}<0} b_{i j} \widetilde{a}_{j} \ominus \sum_{c_{i j} \geq 0} c_{i j} \widetilde{a}_{j} \oplus \sum_{c_{i j}<0} c_{i j} \widetilde{a}_{j}\right)=\widetilde{0},
\end{aligned}
$$

where:

$$
\begin{aligned}
& (A)_{i j}=f\left(t_{j}\right) w\left(x_{d j}\right) w\left(t_{j}\right) s_{i}\left(x_{d j}\right) s_{i}\left(t_{j}\right), \\
& (\widetilde{H})_{i j}=\left(\widetilde{a}_{d} \ominus \widetilde{b}_{d} x_{d j}\right) e^{\widetilde{\lambda}_{d} t_{j}}, \\
& (B)_{i j}=w\left(x_{d j}\right) w\left(t_{j}\right)\left(\left(i+\frac{1}{2}\right) u_{i}(t)-\frac{1}{2} s_{i}\left(t_{j}\right)\right) s_{i}\left(x_{d j}\right), \\
& (C)_{i j}=\left(-w^{\prime}\left(t_{j}\right) w\left(x_{d j}\right)\right) s_{i}\left(x_{d j}\right) s_{i}\left(t_{j}\right) .
\end{aligned}
$$

\subsection{Description of the fuzzy collocation method}

In order to obtain the approximation solution of Eq. (1), according to the fuzzy collocation method [20], we can write this equation as follows:

$$
\widetilde{u}\left(x_{d}, t\right) \approx \widetilde{u}_{n}\left(x_{d}, t\right)=\sum_{i=0}^{n} \widetilde{a}_{i} h_{i}\left(x_{d}\right) h_{i}(t)
$$

where $h_{j}\left(x_{d}\right)$ and $h_{j}(t)(j=0,1, \cdots, n)$ are orthogonal functions.

The collocation condition is as follows:

$$
\widetilde{R}_{n}\left(x_{d j}, t_{j}\right)=\widetilde{0}
$$

It means that:

$$
\underline{R}_{n}^{r}\left(x_{d j}, t_{j}\right)=0, \quad \bar{R}_{n}^{r}\left(x_{d j}, t_{j}\right)=0, \quad \forall r \in[0,1],
$$

where, $x_{d j}$ and $t_{j}(j=1, \cdots, n)$ are collocation points.

We integral from Eq. (1) with respect to $t$. It should be noted that $\sum_{i=1}^{n} \widetilde{a}_{i} h_{i}\left(x_{d}\right) h_{i}(t)$ is $(i)$ differentiable.

$$
\begin{aligned}
\widetilde{u}\left(x_{d}, t\right)= & \ominus \int_{0}^{t} f(u) \widetilde{u}\left(x_{d}, u\right) d u \\
& \ominus \int_{0}^{t}\left(\widetilde{a}_{d} \ominus \widetilde{b}_{d} x_{d}\right) e^{\widetilde{\lambda}_{d} u} d u .
\end{aligned}
$$




$$
\begin{aligned}
& \ominus \int_{0}^{t} f(u) \widetilde{u}_{n}\left(x_{d}, u\right) d u \ominus \int_{0}^{t}\left(\widetilde{a}_{d} \ominus \widetilde{b}_{d} x_{d}\right) e^{\tilde{\lambda}_{d} u} d u \\
& \quad=\widetilde{u}_{n}\left(x_{d}, t\right) \oplus \widetilde{R}_{n}\left(x_{d}, t\right), \\
& \ominus \int_{0}^{t} f(u) \widetilde{u}_{n}\left(x_{d}, u\right) d u \ominus \int_{0}^{t}\left(\widetilde{a}_{d} \ominus \widetilde{b}_{d} x_{d}\right) e^{\widetilde{\lambda}_{d} u} d u \\
& \ominus \widetilde{u}_{n}\left(x_{d}, t\right)=\widetilde{R}_{n}\left(x_{d}, t\right) .
\end{aligned}
$$

According to the fast collocation condition, Eq. (43) can be written in the following operator form:

$$
\begin{aligned}
& \ominus A \widetilde{a} \ominus \widetilde{H} \ominus B \widetilde{a}=\widetilde{0} \\
& \ominus\left(\sum_{a_{i j} \geq 0} a_{i j} \widetilde{a}_{j} \oplus \sum_{a_{i j}<0} a_{i j} \widetilde{a}_{j}\right) \ominus \widetilde{h}_{i j} \\
& \ominus\left(\sum_{b_{i j} \geq 0} b_{i j} \tilde{a}_{j} \oplus \sum_{b_{i j}<0} b_{i j} \widetilde{a}_{j}\right)=\widetilde{0}
\end{aligned}
$$

where:

$$
\begin{aligned}
& (\widetilde{H})_{i j}=\int_{0}^{t_{j}}\left(\widetilde{a}_{d} \ominus \widetilde{b}_{d} x_{d j}\right) e^{\widetilde{\lambda}_{d} u} d u, \\
& (A)_{i j}=\int_{0}^{t_{j}} f(u) h_{i}(u) h_{j}\left(x_{d j}\right) d t, \\
& (B)_{i j}=h_{i}\left(x_{d j}\right) h_{i}\left(t_{j}\right) .
\end{aligned}
$$

We integral from Eq.(1) with respect to $t$. It should be noted that $\sum_{i=0}^{n} \widetilde{a}_{i} h_{i}\left(x_{d}\right) h_{i}(t)$ is (ii)-differentiable:

$$
\begin{gathered}
\widetilde{u}\left(x_{d}, t\right)=\ominus\left(\ominus \int_{0}^{t} f(u) \widetilde{u}\left(x_{d}, u\right) d u\right. \\
\left.\ominus \int_{0}^{t}\left(\widetilde{a}_{d} \ominus \widetilde{b}_{d} x_{d}\right) e^{\widetilde{\lambda}_{d} u} d u\right), \\
\ominus\left(\ominus \int_{0}^{t} f(u) \widetilde{u}_{n}\left(x_{d}, u\right) d u \ominus \int_{0}^{t}\left(\widetilde{a}_{d} \ominus \widetilde{b}_{d} x_{d}\right) e^{\tilde{\lambda}_{d} u} d u\right) \\
=\widetilde{u}_{n}\left(x_{d}, t\right) \oplus \widetilde{R}_{n}\left(x_{d}, t\right), \\
\ominus\left(\ominus \int_{0}^{t} f(u) \widetilde{u}_{n}\left(x_{d}, u\right) d u \ominus \int_{0}^{t}\left(\widetilde{a}_{d} \ominus \widetilde{b}_{d} x_{d}\right) e^{\widetilde{\lambda}_{d} u} d u\right) \\
\ominus \widetilde{u}_{n}\left(x_{d}, t\right)=\widetilde{R}_{n}\left(x_{d}, t\right) .
\end{gathered}
$$

According to the fast collocation condition, Eq. (48) can be written in the following operator form:

$$
\begin{gathered}
\ominus(\ominus A \widetilde{a} \ominus \widetilde{H}) \ominus B \widetilde{a}=\widetilde{0} \\
\ominus\left(\ominus\left(\sum_{a_{i j} \geq 0} a_{i j} \widetilde{a}_{j} \oplus \sum_{a_{i j}<0} a_{i j} \widetilde{a}_{j}\right) \ominus \widetilde{h}_{i j}\right) \\
\ominus\left(\sum_{b_{i j} \geq 0} b_{i j} \widetilde{a}_{j} \oplus \sum_{b_{i j}<0} b_{i j} \widetilde{a}_{j}\right)=\widetilde{0}
\end{gathered}
$$

where:

$$
\begin{aligned}
& (\widetilde{H})_{i j}=\int_{0}^{t_{j}}\left(\widetilde{a}_{d} \ominus \widetilde{b}_{d} x_{d j}\right) e^{\widetilde{\lambda}_{d} u} d u, \\
& (A)_{i j}=\int_{0}^{t_{j}} f(u) h_{i}(u) h_{j}\left(x_{d j}\right) d t, \\
& (B)_{i j}=h_{i}\left(x_{d j}\right) h_{i}\left(t_{j}\right) .
\end{aligned}
$$

\subsection{Existence and convergence analysis}

In this section, we are going to prove the existence and uniqueness of the solution and convergence of the proposed methods by using the following assumptions:

$$
\begin{aligned}
& |f(t)| \leq M_{1}, \\
& D\left(\left(\widetilde{a}_{d} \ominus \widetilde{b}_{d} x_{d}\right) e^{\widetilde{\lambda}_{d} x_{d}}, \widetilde{0}\right) \leq M_{2} .
\end{aligned}
$$

Let:

$$
\alpha_{1}=T\left(M_{1}+M_{2}\right)
$$

According to Eqs. (12) and (28), we have $\underline{R}_{n}^{r}=\bar{R}_{n}^{r}=0$, so, $D\left(\widetilde{R}_{n}, \widetilde{0}\right) \rightarrow 0$. Therefore, we can write:

$$
D\left(\widetilde{a}, \widetilde{a}^{(n)}\right) \leq c_{1} n^{-z}, \quad z>1
$$

Lemma 1: If $\widetilde{u}, \widetilde{v}, \widetilde{w} \in E^{n}$ and $\lambda \in R$, then:

(i) $D(\widetilde{u} \ominus \widetilde{v}, \widetilde{u} \ominus \widetilde{w})=D(\widetilde{v}, \widetilde{w})$,

(ii) $D(\ominus \lambda \widetilde{u}, \ominus \lambda \widetilde{v})=|\lambda| D(\widetilde{u}, \widetilde{v})$.

The proof of this Lemma is provided by [21].

Theorem 1. Let $0<\alpha_{1}<1$; then, Problem (1) has a unique solution when $\widetilde{u}^{\prime}\left(x_{d}, t\right)$ is $(i i)$-differentiable. 
Proof. Let $\widetilde{u}\left(x_{d}, t\right)$ and $\widetilde{u}^{*}\left(x_{d}, t\right)$ be two different solutions of Problem (1); then:

$$
\begin{aligned}
D & \left(\widetilde{u}\left(x_{d}, t\right), \widetilde{u}^{*}\left(x_{d}, t\right)\right) \\
& =D\left(\ominus\left(\ominus \int_{0}^{t} f(u) \widetilde{u}\left(x_{d}, u\right) d u \ominus \int_{0}^{t}\left(\widetilde{a}_{d} \ominus \widetilde{b}_{d} x_{d}\right) e^{\widetilde{\lambda}_{d} u} d u\right),\right. \\
& \left.\ominus\left(\ominus \int_{0}^{t} f(u) \widetilde{u}^{*}\left(x_{d}, u\right) d u \ominus \int_{0}^{t}\left(\widetilde{a}_{d} \ominus \widetilde{b}_{d} x_{d}\right) e^{\widetilde{\lambda}_{d} u} d u\right)\right) \\
& =D\left(\ominus \left(\ominus \int_{0}^{t} f(u) \widetilde{u}\left(x_{d}, u\right) d u,\right.\right. \\
& \ominus\left(\ominus \int_{0}^{t} f(u) \widetilde{u}^{*}\left(x_{d}, u\right) d u\right) \\
& \leq T\left(M_{1}+M_{2}\right) D\left(\widetilde{u}\left(x_{d}, t\right), \widetilde{u}^{*}\left(x_{d}, t\right)\right) \\
& =\alpha_{1} D\left(\widetilde{u}\left(x_{d}, t\right), \widetilde{u}^{*}\left(x_{d}, t\right)\right),
\end{aligned}
$$

from which we get $\left(1-\alpha_{1}\right) D\left(\widetilde{u}\left(x_{d}, t\right), \widetilde{u}^{*}(x, t)\right) \leq 0$. Since $0<\alpha_{1}<1$, then $D\left(\widetilde{u}\left(x_{d}, t\right), \widetilde{u}^{*}\left(x_{d}, t\right)\right)=0$. Hence, implies that $\widetilde{u}\left(x_{d}, t\right)=\widetilde{u}^{*}\left(x_{d}, t\right)$, so Problem (1) has a unique solution.

Theorem 2. Suppose that:

$$
\begin{array}{ll}
\int_{0}^{t}|f(u)| d u \leq L_{1}, & L_{1}<1, \\
\left.\int_{0}^{t} D\left(\left(\widetilde{a}_{d} \ominus \widetilde{b}_{d} x_{d}\right) e^{\widetilde{\widetilde{d}}_{d} u} d u\right), \widetilde{0}\right) \leq L_{2}, & L_{2}<1 .
\end{array}
$$

Then, the solution of Problem (1) is bounded.

Proof. Let $\widetilde{u}\left(x_{d}, t\right)$ be an unbounded solution of Problem (1). Then, for every $r>0$, there exists an element $x_{1 d}$ and $t_{1} \in[0, T]$ such that:

$$
\begin{array}{ll}
D\left(\widetilde{u}\left(x_{d}, t_{1}\right), \widetilde{0}\right)<r, \quad \forall x \in\left[0, x_{1 d}\right], \quad \forall t \in\left[0, t_{1}\right], \\
D\left(\widetilde{u}\left(x_{1 d}, t_{1}\right), \widetilde{0}\right)=r .
\end{array}
$$

Clearly, we can find a positive number $r$ with:

$$
L_{1}+r L_{2}<r .
$$

By Relations (51) and (52) and the assumptions of the theorem, we have:

$$
\begin{aligned}
r & =D\left(\widetilde{u}\left(x_{1 d}, t_{1}\right), \widetilde{0}\right)=D\left(\ominus \left(\ominus \int_{0}^{t_{1}} f(u) \widetilde{u}\left(x_{1 d}, u\right) d u\right.\right. \\
& \left.\left.\ominus \int_{0}^{t_{1}}\left(\widetilde{a}_{d} \ominus \widetilde{b}_{d} x_{1 d}\right) e^{\widetilde{\lambda}_{d} u} d u\right), \widetilde{0}\right) \\
& \leq \int_{0}^{t_{1}}|f(u)| D\left(u\left(x_{1 d}, u\right), \widetilde{0}\right) d u \\
& \left.+\int_{0}^{t_{1}} D\left(\left(\widetilde{a}_{d} \ominus \widetilde{b}_{d} x_{1 d}\right) e^{\widetilde{\lambda}_{d} u} d u\right), \widetilde{0}\right) \leq L_{1}+r L_{2}<r,
\end{aligned}
$$

which is a contraction. Thus, $u\left(x_{d}, t\right)$ is bounded. Therefore, we can write $D\left(\widetilde{a}^{(n)}, \widetilde{0}\right) \leq c_{2} \mathrm{~J}^{-z}, z>1$.

Remark 1. The proof of other cases is similar to that of the previous theorems.

Theorem 3. The maximum absolute truncation error of the solution $\widetilde{u}_{n}\left(x_{d}, t\right)=\sum_{j=0}^{n} \widetilde{a}_{j} h_{j}\left(x_{d}\right) h_{j}(t)$ for Problem (1) by using the Jacobi polynomials method is estimated to be:

$$
D\left(\widetilde{E}_{n}\left(x_{d}, t\right), \widetilde{0}\right) \leq c_{1} \frac{n^{-z+1}}{1-z}+c_{2} n^{-z+1}
$$

Proof. We have:

$$
\begin{aligned}
D( & \left.\widetilde{u}\left(x_{d}, t\right), \widetilde{u}_{n}\left(x_{d}, t\right)\right) \\
& =D\left(\sum_{j=0}^{\infty} \widetilde{a}_{j} p_{j}^{\alpha, \beta}\left(x_{d}\right) p_{j}^{\alpha, \beta}(t), \sum_{j=0}^{n} \widetilde{a}_{j}^{(n)} p_{j}^{\alpha, \beta}\left(x_{d}\right) p_{j}^{\alpha, \beta}(t)\right) \\
& =D\left(\sum_{j=0}^{n} \widetilde{a}_{j} p_{j}^{\alpha, \beta}\left(x_{d}\right) p_{j}^{\alpha, \beta}(t)\right. \\
& \left.+\sum_{j=0}^{n} \widetilde{a}_{j}^{(n)} p_{j}^{\alpha, \beta}\left(x_{d}\right) p_{j}^{\alpha, \beta}(t), \sum_{j=n+1}^{\infty} \widetilde{a}_{j} p_{j}^{\alpha, \beta}\left(x_{d}\right) p_{j}^{\alpha, \beta}(t)\right) \\
& \leq D\left(\sum_{j=0}^{n} \widetilde{a}_{j} p_{j}^{\alpha, \beta}\left(x_{d}\right) p_{j}^{\alpha, \beta}(t), \sum_{j=0}^{n} \widetilde{a}_{j}^{(n)} p_{j}^{\alpha, \beta}\left(x_{d}\right) p_{j}^{\alpha, \beta}(t)\right) \\
& +D\left(\sum_{j=n+1}^{\infty} \widetilde{a}_{j} p_{j}^{\alpha, \beta}\left(x_{d}\right) p_{j}^{\alpha, \beta}(t), \widetilde{0}^{\alpha}\right) \\
& \leq \sum_{j=0}^{n}\left|p_{j}^{\alpha, \beta}\left(x_{d}\right) p_{j}^{\alpha, \beta}(t)\right| D\left(\widetilde{a}_{j}, \widetilde{a}_{j}^{(n)}\right)
\end{aligned}
$$




$$
\begin{aligned}
& +\sum_{j=n+1}^{\infty}\left|p_{j}^{\alpha, \beta}\left(x_{d}\right) p_{j}^{\alpha, \beta}(t)\right| D\left(\widetilde{a}_{j}, \widetilde{0}\right) \\
& \leq \sum_{j=0}^{n} D\left(\widetilde{a}_{j}, \widetilde{a}_{j}^{(n)}\right)+\sum_{j=n+1}^{\infty} D\left(\widetilde{a}_{j}, \widetilde{0}\right) \\
& \leq c_{1} \frac{n^{-z+1}}{1-z}+c_{2} n^{-z+1} .
\end{aligned}
$$

Remark 2. The maximum absolute truncation error of the solution $\widetilde{u}_{n}\left(x_{d}, t\right)=w\left(x_{d}\right) w(t) \sum_{i=0}^{n} \widetilde{a}_{i} p_{j}^{\alpha, \beta}$ $\left(x_{d}\right) p_{j}^{\alpha, \beta}(t)$ to Problem (1) by using the collocation method is similar to that in the Jacobi polynomials method.

Based on the obtained results from the proposed fuzzy collocation methods and with respect to Eq. (3), the optimal retail price that maximizes the discount profit of retailer over period $\left[z_{i-1}, z_{i}\right]$ can be calculated by taking the $(i)$ - and (ii)-differentiables with respect to $x_{d}$ and setting the results equal to zero as follows:

Case (1): If $\tilde{\pi}\left(x_{d}, t\right)_{R}$ is $(i)$-differentiable, then:

$$
\begin{gathered}
\frac{\partial}{\partial x_{d}} \tilde{\pi}\left(x_{d}, t\right)_{R}=\sum_{j=z_{k-1}}^{z_{k}} e^{-R t_{j}}\left(\widetilde{a} \ominus \widetilde{b} x_{d}\right) e^{\lambda t_{j}} \\
\ominus \widetilde{b} e^{\lambda t_{j}} x_{d} e^{-R t_{j}} \ominus \widetilde{w}_{d} e^{-R z_{k-1}} \sum_{i=0}^{n} \widetilde{a}_{i} p_{i}^{\alpha, \beta}\left(x_{d}\right) \\
p_{i}^{\alpha, \beta}\left(z_{k-1}\right) \frac{1}{2}(i+\alpha+\beta+1) p_{i-1}^{(\alpha+1, \beta+1)}\left(x_{d}\right) \\
\ominus \sum_{j=z_{k-1}}^{z_{k}} \widetilde{h}_{d} e^{-R t_{j}} \sum_{i=0}^{n} \widetilde{a}_{i} \frac{1}{2}(i+\alpha+\beta+1) \\
p_{i-1}^{(\alpha+1, \beta+1)}\left(x_{d}\right) p_{i}^{\alpha, \beta}(t) \\
\ominus \widetilde{A}_{R_{d}} e^{-R z_{k-1}} \sum_{i=0}^{n} \widetilde{a}_{i} \frac{1}{2}(i+\alpha+\beta+1) \\
p_{i-1}^{(\alpha+1, \beta+1)}\left(x_{d}\right) p_{i}^{\alpha, \beta}\left(z_{k-1}\right)=\widetilde{0} .
\end{gathered}
$$

Case (2): If $\tilde{\pi}\left(x_{d}, t\right)_{R}$ is (ii)-differentiable, then:

$$
\begin{aligned}
& \frac{\partial}{\partial x_{d}} \widetilde{\pi}\left(x_{d}, t\right)_{R}=\sum_{j=z_{k-1}}^{z_{k}} e^{-R t_{j}}\left(\widetilde{a} \ominus \widetilde{b} x_{d}\right) e^{\lambda t_{j}} \\
& +\widetilde{b} e^{\lambda t_{j}} x_{d} e^{-R t_{j}} \oplus \widetilde{w}_{d} e^{-R z_{k-1}} \sum_{i=0}^{n} \widetilde{a}_{i} p_{i}^{\alpha, \beta}\left(x_{d}\right) \\
& p_{i}^{\alpha, \beta}\left(z_{k-1}\right) \frac{1}{2}(i+\alpha+\beta+1) p_{i-1}^{(\alpha+1, \beta+1)}\left(x_{d}\right)
\end{aligned}
$$

$$
\begin{gathered}
\oplus \sum_{j=z_{k-1}}^{z_{k}} \widetilde{h}_{d} e^{-R t_{j}} \sum_{i=0}^{n} \widetilde{a}_{i} \frac{1}{2}(i+\alpha+\beta+1) \\
p_{i-1}^{(\alpha+1, \beta+1)}\left(x_{d}\right) p_{i}^{\alpha, \beta}(t) \\
\oplus \widetilde{A}_{R_{d}} e^{-R z_{k-1}} \sum_{i=0}^{n} \widetilde{a}_{i} \frac{1}{2}(i+\alpha+\beta+1) \\
p_{i-1}^{(\alpha+1, \beta+1)}\left(x_{d}\right) p_{i}^{\alpha, \beta}\left(z_{k-1}\right)=\widetilde{0} \\
\widetilde{\pi}\left(x_{d}, t\right)_{M}=\widetilde{w}_{d} e^{-R z_{k-1}} \sum_{i=0}^{n} \widetilde{a}_{i} p_{i}^{\alpha, \beta}\left(x_{d}\right) p_{i}^{\alpha, \beta}\left(z_{k-1}\right) \\
\ominus \widetilde{c}_{d} e^{-R z_{k-1}} \sum_{i=0}^{n} \widetilde{a}_{i} p_{i}^{\alpha, \beta}\left(x_{d}\right) p_{i}^{\alpha, \beta}\left(z_{k-1}\right) \\
\ominus \widetilde{A}_{M_{d}} e^{-R z_{k-1}} \sum_{i=0}^{n} \widetilde{a}_{i} p_{i}^{\alpha, \beta}\left(x_{d}\right) p_{i}^{\alpha, \beta}\left(z_{k-1}\right) \\
\ominus \widetilde{S}_{M_{d}} e^{-R z_{k-1}} \cdot
\end{gathered}
$$

Replacing the variable $x_{d}$ by the optimal value of this variable, $x_{d}^{*}$, in Eqs. (2) to (4) yields the replenished order quantity $\left(\widetilde{Q}^{*}\left(x_{d}\right)\right)$, the maximum discount profit of retailer $\tilde{\pi}^{*}\left(x_{d}, t\right)_{R}$, and the maximum discount profit of manufacturer $\widetilde{\pi}^{*}\left(x_{d}, t\right)_{M}$ over an arbitrary period $\left[z_{i-1}, z_{i}\right]$. Moreover, the optimal replenishment schedule in a multi-period planning horizon and associated retail price, the discount profits of the retailer and manufacturer, and replenishment quantity can be calculated by utilizing dynamic programming as follows:

$$
\begin{aligned}
& \tilde{\pi}^{*}\left(x_{d}, t\right)_{R, z_{k}}=\max \left\{\tilde{\pi}^{*}\left(x_{d}, t\right)_{R, z_{k-1}}+\tilde{\pi}^{*}\left(x_{d}, t\right)_{R},\right. \\
& 0 \leq z_{k-1} \leq z_{k} \leq T \\
& \widetilde{Q}^{*}\left(x_{d}\right)=\widetilde{u}\left(x_{d}^{*}, z_{k-1}\right), \\
& \tilde{\pi}^{*}\left(x_{d}, t\right)_{R}=\tilde{\pi}\left(x_{d}^{*}, t\right)_{R}, \\
& \tilde{\pi}^{*}\left(x_{d}, t\right)_{M}=\tilde{\pi}\left(x_{d}^{*}, t\right)_{M}, \\
& \tilde{\pi}^{*}\left(x_{d}, t\right)_{R, z_{k}}=\max \left\{\tilde{\pi}^{*}\left(x_{d}, t\right)_{R, z_{k-1}}+\tilde{\pi}^{*}\left(x_{d}, t\right)_{R},\right. \\
& \left.\quad 0 \leq z_{k-1} \leq z_{k} \leq T\right\} .
\end{aligned}
$$

\section{Validation and evaluation of results}

In this section, in order to validate the proposed model and solution approaches, several numerical examples are implemented and the related obtained results are reported. Also, all mathematical models are coded in Mathematica 7 . The settings in this work are 
as follows: number of periods $=15$; number of products: $d=3$; cost parameters for each product: $\quad \widetilde{S}_{M_{1}}=(80,100,120), \quad \widetilde{S}_{M_{2}}=(75,105,119)$, $\widetilde{S}_{M_{3}}=(105,115,135) ; \quad \widetilde{S}_{R_{1}}=(30,50,75), \widetilde{S}_{R_{2}}=$ $(24,57,70), \widetilde{S}_{R_{3}}=(43,65,83) ; \widetilde{A}_{M_{1}}=(0.3,0.5,0.6)$, $\widetilde{A}_{M_{2}}=(0.2,0.6,0.8), \widetilde{A}_{M_{3}}=(0.4,0.5,0.8) ; \widetilde{A}_{R_{1}}=$ $(0.1,0.19,0.23), \quad \widetilde{A}_{R_{2}}=(0.08,0,23,0.31), \quad \widetilde{A}_{R_{3}}=$ $(0.2,0.27,0.38) ; \widetilde{c}_{1}=(0.8,1,1.6), \widetilde{c}_{2}=(0.65,1.2,1.5)$, $\widetilde{c}_{3}=(0.72,0.12,1.8) ; \widetilde{w}_{1}=(3,5,8), \widetilde{w}_{2}=(2,6,8)$, $\widetilde{w}_{3}=(4,7,9) ;$ and $\widetilde{h}_{1}=(0.04,0.06,0.08), \widetilde{h}_{2}=$ $(0.03,0.07,0.09), \widetilde{h}_{3}=(0.02,0.06,0.1)$; Rate of deterioration for each product: $\tilde{\theta}_{1}=(0.02,0.03,0.05)$, $\tilde{\theta}_{2}=(0.03,0.05,0.07), \tilde{\theta}_{3}=(0.02,0.04,0.06) ;$ demand parameters: $\widetilde{a}_{1}=(430,500,520), \widetilde{a}_{2}=(400,460,500)$, $\widetilde{a}_{3}=(450,510,560) ; \widetilde{b}_{1}=(17,20,25), \widetilde{b}_{2}=(20,25,30)$, $\widetilde{b}_{3}=(16,22,31) ;$ and $\tilde{\lambda}_{1}=(-0.05,-0.08,-0.13)$, $\widetilde{\lambda}_{2}=(-0.06,-0.1,-0.15), \widetilde{\lambda}_{3}=(-0.04,-0.09,-0.11)$.

The summary of test results on the basis of $r=$ $0.5, n=6, \epsilon=10^{-4}, \alpha=-\frac{1}{2}$ and $\beta=-\frac{1}{2}$ for Jacobi polynomials fuzzy collocation method is provided in Tables 1-9. Also, the summary of test results on the basis of $r=0.5, n=8$, and $\epsilon=10^{-4}$ for airfoil polynomials fuzzy collocation method is provided in Tables 10-18. Moreover, the summary of test results on the basis of $r=0.5, n=9$, and $\epsilon=10^{-4}$ for fuzzy collocation method is provided in Tables 19-24. Moreover, the pseudo-code of the proposed solution approaches is given below.

Table 1. Numerical results by using the Jacobi polynomials fuzzy collocation method; Case $1, d=1$.

\begin{tabular}{ccccccc}
\hline $\boldsymbol{k}^{*}\left[\boldsymbol{z}_{\boldsymbol{k}-\mathbf{1}}^{*}, \boldsymbol{z}_{\boldsymbol{k}-\mathbf{1}}^{*}\right]$ & $\left(\underline{\boldsymbol{x}}_{\mathbf{1}}^{*}, \overline{\boldsymbol{x}}_{\mathbf{1}}^{*}\right)$ & $\left(\underline{\boldsymbol{Q}}\left(\boldsymbol{x}_{\mathbf{1}}^{*}\right), \overline{\boldsymbol{Q}}\left(\boldsymbol{x}_{\mathbf{1}}^{*}\right)\right)$ & $\left(\underline{\boldsymbol{\pi}}\left(\boldsymbol{x}_{\mathbf{1}}^{*}\right)_{\boldsymbol{R}}, \overline{\boldsymbol{\pi}}\left(\boldsymbol{x}_{\mathbf{1}}^{*}\right)_{\boldsymbol{R}}\right)$ & $\left(\underline{\boldsymbol{\pi}}\left(\boldsymbol{x}_{\mathbf{1}}^{*}\right)_{M}, \overline{\boldsymbol{\pi}}\left(\boldsymbol{x}_{\mathbf{1}}^{*}\right)_{\boldsymbol{M}}\right)$ & $\left(\underline{\boldsymbol{\pi}}\left(\boldsymbol{x}_{\mathbf{1}}^{*}\right)_{C}, \overline{\boldsymbol{\pi}}\left(\boldsymbol{x}_{\mathbf{1}}^{*}\right)_{C}\right)$ \\
\hline 1 & {$[0,4]$} & $(13.689,13.847)$ & $(554.137,572.308)$ & $(3495.33,3622.56)$ & $(2088.12,2245.63)$ & $(5583.45,5868.19)$ \\
2 & {$[4,7]$} & $(13.776,13.8158)$ & $(276.308,294.205)$ & $(1475.21,1691.47)$ & $(838.85,852.93)$ & $(2314.06,2544.4)$ \\
3 & {$[7,11]$} & $(13.858,14.072)$ & $(270.433,272.509)$ & $(1119.66,1323.32)$ & $(663.518,680.157)$ & $(1783.18,2003.48)$ \\
4 & {$[11,15]$} & $(13.049,15.624)$ & $(178.419,182.739)$ & $(638.275,652.817)$ & $(337.418,356.309)$ & $(975.693,1009.13)$ \\
$\sum$ & & $(54.372,57.3598)$ & $(1279.297,1321.761)$ & $(6728.475,7290.167)$ & $(3927.906,4144.026)$ & $(10656.383,11425.2)$ \\
\hline
\end{tabular}

Table 2. Numerical results by using the Jacobi polynomials fuzzy collocation method; Case $1, d=2$.

\begin{tabular}{ccccccc}
\hline $\boldsymbol{k}^{*}\left[\boldsymbol{z}_{\boldsymbol{k}-\mathbf{1}}^{*}, \boldsymbol{z}_{\boldsymbol{k}-\mathbf{1}}^{*}\right]$ & $\left(\underline{\boldsymbol{x}}_{\mathbf{2}}^{*}, \overline{\boldsymbol{x}}_{\mathbf{2}}^{*}\right)$ & $\left(\underline{\boldsymbol{Q}}\left(\boldsymbol{x}_{\mathbf{2}}^{*}\right), \overline{\boldsymbol{Q}}\left(\boldsymbol{x}_{\mathbf{2}}^{*}\right)\right)$ & $\left(\underline{\boldsymbol{\pi}}\left(\boldsymbol{x}_{\mathbf{2}}^{*}\right)_{\boldsymbol{R}}, \overline{\boldsymbol{\pi}}\left(\boldsymbol{x}_{\mathbf{2}}^{*}\right)_{\boldsymbol{R}}\right)$ & $\left(\underline{\boldsymbol{\pi}}\left(\boldsymbol{x}_{\mathbf{2}}^{*}\right)_{M}, \overline{\boldsymbol{\pi}}\left(\boldsymbol{x}_{\mathbf{2}}^{*}\right)_{\boldsymbol{M}}\right)$ & $\left(\underline{\boldsymbol{\pi}}\left(\boldsymbol{x}_{\mathbf{2}}^{*}\right)_{C}, \overline{\boldsymbol{\pi}}\left(\boldsymbol{x}_{\mathbf{2}}^{*}\right)_{\boldsymbol{C}}\right)$ \\
\hline 1 & {$[0,3]$} & $(13.626,14.085)$ & $(417.34,436.18)$ & $(2744.32,2966.12)$ & $(1486.21,1613.25)$ & $(4230.53,4579.37)$ \\
2 & {$[3,8]$} & $(13.889,14.188)$ & $(475.234,490.54)$ & $(2314.11,2578.45)$ & $(1417.88,1623.49)$ & $(3731.99,4201.94)$ \\
3 & {$[8,12]$} & $(13.908,14.266)$ & $(239.077,241.604)$ & $(997.67,1107,26)$ & $(551.422,579.616)$ & $(1549.092,1686.876)$ \\
4 & {$[12,15]$} & $(13.957,14.341)$ & $(120.487,122.322)$ & $(447.619,460.827)$ & $(1977.355,2114.715)$ & $(2424.974,2575.542)$ \\
$\sum$ & & $(61.38,56.88)$ & $(1252.138,1290.646)$ & $(6503.719,7112.657)$ & $(5432.867,5931.071)$ & $(11936.586,13043.728)$ \\
\hline
\end{tabular}

Table 3. Numerical results by using the Jacobi polynomials fuzzy collocation method; Case $1, d=3$.

\begin{tabular}{ccccccc}
\hline $\boldsymbol{k}^{*}\left[\boldsymbol{z}_{\boldsymbol{k}-\mathbf{1}}^{*}, \boldsymbol{z}_{\boldsymbol{k}-\mathbf{1}}^{*}\right]$ & $\left(\underline{\boldsymbol{x}}_{\mathbf{3}}^{*}, \overline{\boldsymbol{x}}_{\mathbf{3}}^{*}\right)$ & $\left(\underline{\boldsymbol{Q}}\left(\boldsymbol{x}_{\mathbf{3}}^{*}\right), \overline{\boldsymbol{Q}}\left(\boldsymbol{x}_{\mathbf{3}}^{*}\right)\right)$ & $\left(\underline{\boldsymbol{\pi}}\left(\boldsymbol{x}_{\mathbf{3}}^{*}\right)_{\boldsymbol{R}}, \overline{\boldsymbol{\pi}}\left(\boldsymbol{x}_{\mathbf{3}}^{*}\right)_{\boldsymbol{R}}\right)$ & $\left(\underline{\boldsymbol{\pi}}\left(\boldsymbol{x}_{\mathbf{3}}^{*}\right)_{\boldsymbol{M}}, \overline{\boldsymbol{\pi}}\left(\boldsymbol{x}_{\mathbf{3}}^{*}\right)_{\boldsymbol{M}}\right)$ & $\left(\underline{\boldsymbol{\pi}}\left(\boldsymbol{x}_{\mathbf{3}}^{*}\right)_{C}, \overline{\boldsymbol{\pi}}\left(\boldsymbol{x}_{\mathbf{3}}^{*}\right)_{C}\right)$ \\
\hline 1 & {$[0,4]$} & $(13.309,15.288)$ & $(514.066,534.076)$ & $(3342.17,3567.15)$ & $(1865.73,2037.44)$ & $(5207.9,5604.59)$ \\
2 & {$[4,8]$} & $(13.508,15.126)$ & $(337.835,3507.24)$ & $(1733.88,1955.08)$ & $(989.97,1124.65)$ & $(2723.85,3079.73)$ \\
3 & {$[8,15]$} & $(13.529,15.088)$ & $(361.117,371.225)$ & $(1276.47,1439.46)$ & $(897.69,915.33)$ & $(2174.16,2354.79)$ \\
$\sum$ & & $(40.346,45.502)$ & $(1213.018,4412.541)$ & $(6352.52,6961.69)$ & $(3753.39,4077.42)$ & $(10105.91,11039.11)$ \\
\hline
\end{tabular}

Table 4. Numerical results by using the Jacobi polynomials fuzzy collocation method; Case $2, d=1$.

\begin{tabular}{ccccccc}
\hline $\boldsymbol{k}^{*}\left[\boldsymbol{z}_{\boldsymbol{k}-\mathbf{1}}^{*}, \boldsymbol{z}_{\boldsymbol{k}-\mathbf{1}}^{*}\right]$ & $\left(\underline{\boldsymbol{x}}_{\mathbf{1}}^{*}, \overline{\boldsymbol{x}}_{\mathbf{1}}^{*}\right)$ & $\left(\underline{\boldsymbol{Q}}\left(\boldsymbol{x}_{\mathbf{1}}^{*}\right), \overline{\boldsymbol{Q}}\left(\boldsymbol{x}_{\mathbf{1}}^{*}\right)\right)$ & $\left(\underline{\boldsymbol{\pi}}\left(\boldsymbol{x}_{\mathbf{1}}^{*}\right)_{\boldsymbol{R}}, \overline{\boldsymbol{\pi}}\left(\boldsymbol{x}_{\mathbf{1}}^{*}\right)_{\boldsymbol{R}}\right)$ & $\left(\underline{\boldsymbol{\pi}}\left(\boldsymbol{x}_{\mathbf{1}}^{*}\right)_{M}, \overline{\boldsymbol{\pi}}\left(\boldsymbol{x}_{\mathbf{1}}^{*}\right)_{M}\right)$ & $\left(\underline{\boldsymbol{\pi}}\left(\boldsymbol{x}_{\mathbf{1}}^{*}\right)_{C}, \overline{\boldsymbol{\pi}}\left(\boldsymbol{x}_{\mathbf{1}}^{*}\right)_{C}\right)$ \\
\hline 1 & {$[0,4]$} & $(13.643,13.825)$ & $(554.165,572.329)$ & $(3495.67,3622.46)$ & $(2088.28,2245.58)$ & $(5583.95,5868.04)$ \\
2 & {$[4,7]$} & $(13.748,13.8133)$ & $(276.388,294.295)$ & $(1475.43,1691.69)$ & $(838.77,852.58)$ & $(2314.2,2544.27)$ \\
3 & {$[7,11]$} & $(13.843,14.112)$ & $(270.487,272.565)$ & $(1119.32,1323.77)$ & $(663.563,680.205)$ & $(1782.883,2003.975)$ \\
4 & {$[11,15]$} & $(13.086,15.624)$ & $(178.453,182.782)$ & $(638.282,652.845)$ & $(337.429,356.337)$ & $(975.711,1009.182)$ \\
$\sum$ & & $(54.32,57.3743)$ & $(1279.493,1321.971)$ & $(6728.702,7290.765)$ & $(3928.042,4134.702)$ & $(10656.744,11425.467)$ \\
\hline
\end{tabular}


Table 5. Numerical results by using the Jacobi polynomials fuzzy collocation method; Case $2, d=2$.

\begin{tabular}{ccccccc}
\hline $\boldsymbol{k}^{*}\left[\boldsymbol{z}_{\boldsymbol{k}-\mathbf{1}}^{*}, \boldsymbol{z}_{\boldsymbol{k}-\mathbf{1}}^{*}\right]$ & $\left(\underline{\boldsymbol{x}}_{\mathbf{2}}^{*}, \overline{\boldsymbol{x}}_{\mathbf{2}}^{*}\right)$ & $\left(\underline{\boldsymbol{Q}}\left(\boldsymbol{x}_{\mathbf{2}}^{*}\right), \overline{\boldsymbol{Q}}\left(\boldsymbol{x}_{\mathbf{2}}^{*}\right)\right)$ & $\left(\underline{\boldsymbol{\pi}}\left(\boldsymbol{x}_{\mathbf{2}}^{*}\right)_{\boldsymbol{R}}, \overline{\boldsymbol{\pi}}\left(\boldsymbol{x}_{\mathbf{2}}^{*}\right)_{\boldsymbol{R}}\right)$ & $\left(\underline{\boldsymbol{\pi}}\left(\boldsymbol{x}_{\mathbf{2}}^{*}\right)_{M}, \overline{\boldsymbol{\pi}}\left(\boldsymbol{x}_{\mathbf{2}}^{*}\right)_{\boldsymbol{M}}\right)$ & $\left(\underline{\boldsymbol{\pi}}\left(\boldsymbol{x}_{\mathbf{2}}^{*}\right)_{C}, \overline{\boldsymbol{\pi}}\left(\boldsymbol{x}_{\mathbf{2}}^{*}\right)_{C}\right)$ \\
\hline 1 & {$[0,3]$} & $(13.648,14.026)$ & $(417.88,436.26)$ & $(2744.72,2966.87)$ & $(1486.41,1613.08)$ & $(4231.13,4579.45)$ \\
2 & {$[3,8]$} & $(13.817,14.165)$ & $(475.264,490.29)$ & $(2314.36,2578.14)$ & $(1417.54,1623.35)$ & $(3731.9,4201.49)$ \\
3 & {$[8,12]$} & $(13.922,14.237)$ & $(239.038,241.612)$ & $(997.58,1107.43)$ & $(551.471,579.697)$ & $(1549.051,1687.127)$ \\
4 & {$[12,15]$} & $(13.909,14.378)$ & $(120.495,122.317)$ & $(447.623,460.818)$ & $(1977.364,2114.722)$ & $(2424.987,2575.54)$ \\
$\sum$ & & $(55.296,56.806)$ & $(1252.677,1290.478)$ & $(6504.283,7113.258)$ & $(5432.785,5930.849)$ & $(11937.068,13044.107)$ \\
\hline
\end{tabular}

Table 6. Numerical results by using the Jacobi polynomials fuzzy collocation method; Case $2, d=3$.

\begin{tabular}{ccccccc}
\hline $\boldsymbol{k}^{*}\left[\boldsymbol{z}_{\boldsymbol{k}-\mathbf{1}}^{*}, \boldsymbol{z}_{\boldsymbol{k}-\mathbf{1}}^{*}\right]$ & $\left(\underline{\boldsymbol{x}}_{\mathbf{3}}^{*}, \overline{\boldsymbol{x}}_{\mathbf{3}}^{*}\right)$ & $\left(\underline{\boldsymbol{Q}}\left(\boldsymbol{x}_{\mathbf{3}}^{*}\right), \overline{\boldsymbol{Q}}\left(\boldsymbol{x}_{\mathbf{3}}^{*}\right)\right)$ & $\left(\underline{\boldsymbol{\pi}}\left(\boldsymbol{x}_{\mathbf{3}}^{*}\right)_{\boldsymbol{R}}, \overline{\boldsymbol{\pi}}\left(\boldsymbol{x}_{\mathbf{3}}^{*}\right)_{\boldsymbol{R}}\right)$ & $\left(\underline{\boldsymbol{\pi}}\left(\boldsymbol{x}_{\mathbf{3}}^{*}\right)_{\boldsymbol{M}}, \overline{\boldsymbol{\pi}}\left(\boldsymbol{x}_{\mathbf{3}}^{*}\right)_{\boldsymbol{M}}\right)$ & $\left(\underline{\boldsymbol{\pi}}\left(\boldsymbol{x}_{\mathbf{3}}^{*}\right)_{\boldsymbol{C}}, \overline{\boldsymbol{\pi}}\left(\boldsymbol{x}_{\mathbf{3}}^{*}\right)_{\boldsymbol{C}}\right)$ \\
\hline 1 & {$[0,4]$} & $(13.346,15.236)$ & $(514.027,534.066)$ & $(3378.17,3567.79)$ & $(1865.47,2037.55)$ & $(5243.64,5605.34)$ \\
2 & {$[4,8]$} & $(13.517,15.144)$ & $(337.829,3507.62)$ & $(1733.26,1955.89)$ & $(989.15,1124.88)$ & $(2722.41,3080.77)$ \\
3 & {$[8,15]$} & $(13.557,15.032)$ & $(361.136,371.228)$ & $(1276.16,1439.38)$ & $(897.07,915.73)$ & $(2173.23,2355.11)$ \\
$\sum$ & & $(40.42,45.412)$ & $(1212.992,4412.914)$ & $(6387.59,6963.06)$ & $(3751.69,4078.16)$ & $(10139.28,11041.22)$ \\
\hline
\end{tabular}

Table 7. Numerical results by using the Jacobi polynomials fuzzy collocation method; Case $3, d=1$.

\begin{tabular}{ccccccc}
\hline $\boldsymbol{k}^{*}\left[\boldsymbol{z}_{\boldsymbol{k}-\mathbf{1}}^{*}, \boldsymbol{z}_{\boldsymbol{k}-\mathbf{1}}^{*}\right]$ & $\left(\underline{\boldsymbol{x}}_{\mathbf{1}}^{*}, \overline{\boldsymbol{x}}_{\mathbf{1}}^{*}\right)$ & $\left(\underline{\left.\boldsymbol{Q}\left(\boldsymbol{x}_{\mathbf{1}}^{*}\right), \overline{\boldsymbol{Q}}\left(\boldsymbol{x}_{\mathbf{1}}^{*}\right)\right)}\right.$ & $\left(\underline{\boldsymbol{\pi}}\left(\boldsymbol{x}_{\mathbf{1}}^{*}\right)_{\boldsymbol{R}}, \overline{\boldsymbol{\pi}}\left(\boldsymbol{x}_{\mathbf{1}}^{*}\right)_{\boldsymbol{R}}\right)$ & $\left(\underline{\boldsymbol{\pi}}\left(\boldsymbol{x}_{\mathbf{1}}^{*}\right)_{M}, \overline{\boldsymbol{\pi}}\left(\boldsymbol{x}_{\mathbf{1}}^{*}\right)_{\boldsymbol{M}}\right)$ & $\left(\underline{\boldsymbol{\pi}}\left(\boldsymbol{x}_{\mathbf{1}}^{*}\right)_{C}, \overline{\boldsymbol{\pi}}\left(\boldsymbol{x}_{\mathbf{1}}^{*}\right)_{C}\right)$ \\
\hline 1 & {$[0,4]$} & $(13.605,13.825)$ & $(554.602,572.413)$ & $(3495.59,3622.38)$ & $(2088.38,2245.652)$ & $(5536.45,5868.41)$ \\
2 & {$[4,7]$} & $(13.718,13.8077)$ & $(276.559,294.717)$ & $(1475.67,1691.25)$ & $(838.77,852.19)$ & $(2314.27,2544.69)$ \\
3 & {$[7,11]$} & $(13.838,14.208)$ & $(270.249,272.308)$ & $(1119.43,1323.87)$ & $(663.436,680.217)$ & $(1783.49,2003.08)$ \\
4 & {$[11,15]$} & $(13.112,15.688)$ & $(178.822,182.098)$ & $(638.473,652.549)$ & $(337.388,356.261)$ & $(975.452,1009.35)$ \\
$\sum$ & & $(54.273,57.5287)$ & $(1280.232,1321.536)$ & $(6729.163,7290.049)$ & $(3927.974,4134.374)$ & $(10609.662,11425.53)$ \\
\hline
\end{tabular}

Table 8. Numerical results by using the Jacobi polynomials fuzzy collocation method; Case $3, d=2$.

\begin{tabular}{ccccccc}
\hline $\boldsymbol{k}^{*}\left[\boldsymbol{z}_{\boldsymbol{k}-\mathbf{1}}^{*}, \boldsymbol{z}_{\boldsymbol{k}-\mathbf{1}}^{*}\right]$ & $\left(\underline{\boldsymbol{x}}_{\mathbf{2}}^{*}, \overline{\boldsymbol{x}}_{\mathbf{2}}^{*}\right)$ & $\left(\underline{\boldsymbol{Q}}\left(\boldsymbol{x}_{\mathbf{2}}^{*}\right), \overline{\boldsymbol{Q}}\left(\boldsymbol{x}_{\mathbf{2}}^{*}\right)\right)$ & $\left(\underline{\boldsymbol{\pi}}\left(\boldsymbol{x}_{\mathbf{2}}^{*}\right)_{\boldsymbol{R}}, \overline{\boldsymbol{\pi}}\left(\boldsymbol{x}_{\mathbf{2}}^{*}\right)_{\boldsymbol{R}}\right)$ & $\left(\underline{\boldsymbol{\pi}}\left(\boldsymbol{x}_{\mathbf{2}}^{*}\right)_{\boldsymbol{M}}, \overline{\boldsymbol{\pi}}\left(\boldsymbol{x}_{\mathbf{2}}^{*}\right)_{\boldsymbol{M}}\right)$ & $\left(\underline{\boldsymbol{\pi}}\left(\boldsymbol{x}_{\mathbf{2}}^{*}\right)_{\boldsymbol{C}}, \overline{\boldsymbol{\pi}}\left(\boldsymbol{x}_{\mathbf{2}}^{*}\right)_{\boldsymbol{C}}\right)$ \\
\hline 1 & {$[0,3]$} & $(13.617,14.038)$ & $(417.38,436.75)$ & $(2776.32,2966.48)$ & $(1486.67,1613.17)$ & $(4262.99,4579.65)$ \\
2 & {$[3,8]$} & $(13.842,14.157)$ & $(475.283,490.49)$ & $(2314.52,2578.27)$ & $(1417.36,1623.16)$ & $(3731.88,4201.43)$ \\
3 & {$[8,12]$} & $(13.919,14.227)$ & $(239.022,241.678)$ & $(997.81,1107.43)$ & $(551.482,579.694)$ & $(1549.292,1687.124)$ \\
4 & {$[12,15]$} & $(13.936,14.366)$ & $(120.453,122.339)$ & $(447.605,460.898)$ & $(1977.311,2114.723)$ & $(2424.916,2475.621)$ \\
$\Sigma$ & & $(55.314,56.788)$ & $(1252.138,1291.257)$ & $(6536.255,7113.078)$ & $(5432.823,5930.747)$ & $(11969.078,13043.825)$ \\
\hline
\end{tabular}

Table 9. Numerical results by using the Jacobi polynomials fuzzy collocation method; Case $3, d=3$.

\begin{tabular}{ccccccc}
\hline $\boldsymbol{k}^{*}\left[\boldsymbol{z}_{\boldsymbol{k}-\mathbf{1}}^{*}, \boldsymbol{z}_{\boldsymbol{k}-\mathbf{1}}^{*}\right]$ & $\left(\underline{\boldsymbol{x}}_{\mathbf{3}}^{*}, \overline{\boldsymbol{x}}_{\mathbf{3}}^{*}\right)$ & $\left(\underline{\boldsymbol{Q}}\left(\boldsymbol{x}_{\mathbf{3}}^{*}\right), \overline{\boldsymbol{Q}}\left(\boldsymbol{x}_{\mathbf{3}}^{*}\right)\right)$ & $\left(\underline{\boldsymbol{\pi}}\left(\boldsymbol{x}_{\mathbf{3}}^{*}\right)_{\boldsymbol{R}}, \overline{\boldsymbol{\pi}}\left(\boldsymbol{x}_{\mathbf{3}}^{*}\right)_{\boldsymbol{R}}\right)$ & $\left(\underline{\boldsymbol{\pi}}\left(\boldsymbol{x}_{\mathbf{3}}^{*}\right)_{\boldsymbol{M}}, \overline{\boldsymbol{\pi}}\left(\boldsymbol{x}_{\mathbf{3}}^{*}\right)_{M}\right)$ & $\left(\underline{\boldsymbol{\pi}}\left(\boldsymbol{x}_{\mathbf{3}}^{*}\right)_{C}, \overline{\boldsymbol{\pi}}\left(\boldsymbol{x}_{\mathbf{3}}^{*}\right)_{\boldsymbol{C}}\right)$ \\
\hline 1 & {$[0,4]$} & $(13.373,15.235)$ & $(514.046,534.037)$ & $(3325.17,3567.48)$ & $(1865.77,2037.83)$ & $(5190.94,5605.31)$ \\
2 & {$[4,8]$} & $(13.529,15.162)$ & $(337.853,3507.88)$ & $(1767.26,1955.18)$ & $(989.27,1124.58)$ & $(2756.53,3079.76)$ \\
3 & {$[8,15]$} & $(13.575,15.034)$ & $(361.189,371.252)$ & $(1285.16,1439.59)$ & $(897.18,915.66)$ & $(2182.34,2355.25)$ \\
$\sum$ & & $(40.477,45.431)$ & $(1213.088,4413.169)$ & $(6377.59,6962.25)$ & $(3752.22,4078.07)$ & $(10129.81,11040.32)$ \\
\hline
\end{tabular}

Table 10. Numerical results by using the airfoil polynomials fuzzy collocation method; Case $1, d=1$.

\begin{tabular}{ccccccc}
\hline $\boldsymbol{k}^{*}\left[\boldsymbol{z}_{\boldsymbol{k}-\mathbf{1}}^{*}, \boldsymbol{z}_{\boldsymbol{k}-\mathbf{1}}^{*}\right]$ & $\left(\underline{\boldsymbol{x}}_{\mathbf{1}}^{*}, \overline{\boldsymbol{x}}_{\mathbf{1}}^{*}\right)$ & $\left(\underline{\boldsymbol{Q}}\left(\boldsymbol{x}_{\mathbf{1}}^{*}\right), \overline{\boldsymbol{Q}}\left(\boldsymbol{x}_{\mathbf{1}}^{*}\right)\right)$ & $\left(\underline{\boldsymbol{\pi}}\left(\boldsymbol{x}_{\mathbf{1}}^{*}\right)_{\boldsymbol{R}}, \overline{\boldsymbol{\pi}}\left(\boldsymbol{x}_{\mathbf{1}}^{*}\right)_{\boldsymbol{R}}\right)$ & $\left(\underline{\boldsymbol{\pi}}\left(\boldsymbol{x}_{\mathbf{1}}^{*}\right)_{M}, \overline{\boldsymbol{\pi}}\left(\boldsymbol{x}_{\mathbf{1}}^{*}\right)_{\boldsymbol{M}}\right)$ & $\left(\underline{\boldsymbol{\pi}}\left(\boldsymbol{x}_{\mathbf{1}}^{*}\right)_{\boldsymbol{C}}, \overline{\boldsymbol{\pi}}\left(\boldsymbol{x}_{\mathbf{1}}^{*}\right)_{\boldsymbol{C}}\right)$ \\
\hline 1 & {$[0,4]$} & $(13.673,13.817)$ & $(554.126,572.316)$ & $(3495.65,3622.09)$ & $(2088.19,2245.53)$ & $(5583.84,5867.62)$ \\
2 & {$[4,7]$} & $(13.722,13.8146)$ & $(276.355,294.238)$ & $(1475.42,1691.66)$ & $(838.75,852.28)$ & $(2314.17,2543.94)$ \\
3 & {$[7,11]$} & $(13.839,14.067)$ & $(270.478,272.534)$ & $(1119.71,1323.82)$ & $(663.577,680.187)$ & $(1783.287,2004.007)$ \\
4 & {$[11,15]$} & $(13.082,15.663)$ & $(178.481,182.724)$ & $(638.268,652.822)$ & $(337.463,356.326)$ & $(975.731,1009.148)$ \\
$\sum$ & & $(54.316,57.3616)$ & $(1279.44,1321.812)$ & $(6729.048,7290.392)$ & $(3927.98,4134.323)$ & $(10657.028,11424.715)$ \\
\hline
\end{tabular}


Table 11. Numerical results by using the airfoil polynomials fuzzy collocation method; Case $1, d=2$.

\begin{tabular}{ccccccc}
\hline $\boldsymbol{k}^{*}\left[\boldsymbol{z}_{\boldsymbol{k}-\mathbf{1}}^{*}, \boldsymbol{z}_{\boldsymbol{k}-\mathbf{1}}^{*}\right]$ & $\left(\underline{\boldsymbol{x}}_{2}^{*}, \overline{\boldsymbol{x}}_{\mathbf{2}}^{*}\right)$ & $\left(\underline{\boldsymbol{Q}}\left(\boldsymbol{x}_{\mathbf{2}}^{*}\right), \overline{\boldsymbol{Q}}\left(\boldsymbol{x}_{\mathbf{2}}^{*}\right)\right)$ & $\left(\underline{\boldsymbol{\pi}}\left(\boldsymbol{x}_{\mathbf{2}}^{*}\right)_{\boldsymbol{R}}, \overline{\boldsymbol{\pi}}\left(\boldsymbol{x}_{\mathbf{2}}^{*}\right)_{\boldsymbol{R}}\right)$ & $\left(\underline{\boldsymbol{\pi}}\left(\boldsymbol{x}_{\mathbf{2}}^{*}\right)_{M}, \overline{\boldsymbol{\pi}}\left(\boldsymbol{x}_{\mathbf{2}}^{*}\right)_{\boldsymbol{M}}\right)$ & $\left(\underline{\boldsymbol{\pi}}\left(\boldsymbol{x}_{\mathbf{2}}^{*}\right)_{C}, \overline{\boldsymbol{\pi}}\left(\boldsymbol{x}_{\mathbf{2}}^{*}\right)_{C}\right)$ \\
\hline 1 & {$[0,3]$} & $(13.642,14.067)$ & $(417.12,436.35)$ & $(2744.84,2966.29)$ & $(1486.13,1613.18)$ & $(4230.97,4579.47)$ \\
2 & {$[3,8]$} & $(13.823,14.114)$ & $(475.244,490.71)$ & $(2314.33,2578.65)$ & $(1417.57,1623.27)$ & $(3731.9,4201.92)$ \\
3 & {$[8,12]$} & $(13.972,14.228)$ & $(239.024,241.617)$ & $(997.98,1107.41)$ & $(551.407,579.662)$ & $(1549.387,1687.072)$ \\
4 & {$[12,15]$} & $(13.939,14.326)$ & $(120.412,122.309)$ & $(447.638,460.821)$ & $(1977.355,2114.793)$ & $(2424.993,2575.614)$ \\
$\sum$ & & $(55.367,56.735)$ & $(1251.8,1290.986)$ & $(6504.788,7113.171)$ & $(5432.462,5930.905)$ & $(11937.25,13044.076)$ \\
\hline
\end{tabular}

Table 12. Numerical results by using the airfoil polynomials fuzzy collocation method; Case $1, d=3$.

\begin{tabular}{ccccccc}
\hline $\boldsymbol{k}^{*}\left[\boldsymbol{z}_{\boldsymbol{k}-\mathbf{1}}^{*}, \boldsymbol{z}_{\boldsymbol{k}-\mathbf{1}}^{*}\right]$ & $\left(\underline{\boldsymbol{x}}_{\mathbf{3}}^{*}, \overline{\boldsymbol{x}}_{\mathbf{3}}^{*}\right)$ & $\left(\underline{\boldsymbol{Q}}\left(\boldsymbol{x}_{\mathbf{3}}^{*}\right), \overline{\boldsymbol{Q}}\left(\boldsymbol{x}_{\mathbf{3}}^{*}\right)\right)$ & $\left(\underline{\boldsymbol{\pi}}\left(\boldsymbol{x}_{\mathbf{3}}^{*}\right)_{\boldsymbol{R}}, \overline{\boldsymbol{\pi}}\left(\boldsymbol{x}_{\mathbf{3}}^{*}\right)_{\boldsymbol{R}}\right)$ & $\left(\underline{\boldsymbol{\pi}}\left(\boldsymbol{x}_{\mathbf{3}}^{*}\right)_{\boldsymbol{M}}, \overline{\boldsymbol{\pi}}\left(\boldsymbol{x}_{\mathbf{3}}^{*}\right)_{\boldsymbol{M}}\right)$ & $\left(\underline{\boldsymbol{\pi}}\left(\boldsymbol{x}_{\mathbf{3}}^{*}\right)_{\boldsymbol{C}}, \overline{\boldsymbol{\pi}}\left(\boldsymbol{x}_{\mathbf{3}}^{*}\right)_{\boldsymbol{C}}\right)$ \\
\hline 1 & {$[0,4]$} & $(13.365,15.229)$ & $(514.053,534.022)$ & $(3342.56,3567.41)$ & $(1865.68,2037.79)$ & $(5208.24,5605.2)$ \\
2 & {$[4,8]$} & $(13.544,15.165)$ & $(337.848,3507.63)$ & $(1733.72,1955.16)$ & $(989.45,1124.23)$ & $(2723.17,3079.39)$ \\
3 & {$[8,15]$} & $(13.533,15.026)$ & $(361.122,371.237)$ & $(1276.28,1439.83)$ & $(897.12,915.85)$ & $(2164.4,2355.68)$ \\
$\sum$ & & $(40.442,45.42)$ & $(1213.023,4412.889)$ & $(6352.56,6962.4)$ & $(3752.25,4077.87)$ & $(10095.81,11040.27)$ \\
\hline
\end{tabular}

Table 13. Numerical results by using the airfoil polynomials fuzzy collocation method; Case $2, d=1$.

\begin{tabular}{ccccccc}
\hline $\boldsymbol{k}^{*}\left[\boldsymbol{z}_{\boldsymbol{k}-\mathbf{1}}^{*}, \boldsymbol{z}_{\boldsymbol{k}-\mathbf{1}}^{*}\right]$ & $\left(\underline{\boldsymbol{x}}_{\mathbf{1}}^{*}, \overline{\boldsymbol{x}}_{\mathbf{1}}^{*}\right)$ & $\left(\underline{\boldsymbol{Q}}\left(\boldsymbol{x}_{\mathbf{1}}^{*}\right), \overline{\boldsymbol{Q}}\left(\boldsymbol{x}_{\mathbf{1}}^{*}\right)\right)$ & $\left(\underline{\boldsymbol{\pi}}\left(\boldsymbol{x}_{\mathbf{1}}^{*}\right)_{\boldsymbol{R}}, \overline{\boldsymbol{\pi}}\left(\boldsymbol{x}_{\mathbf{1}}^{*}\right)_{\boldsymbol{R}}\right)$ & $\left(\underline{\boldsymbol{\pi}}\left(\boldsymbol{x}_{\mathbf{1}}^{*}\right)_{M}, \overline{\boldsymbol{\pi}}\left(\boldsymbol{x}_{\mathbf{1}}^{*}\right)_{M}\right)$ & $\left(\underline{\boldsymbol{\pi}}\left(\boldsymbol{x}_{\mathbf{1}}^{*}\right)_{C}, \overline{\boldsymbol{\pi}}\left(\boldsymbol{x}_{\mathbf{1}}^{*}\right)_{C}\right)$ \\
\hline 1 & {$[0,4]$} & $(13.652,13.837)$ & $(554.147,572.311)$ & $(3495.12,3622.66)$ & $(2088.72,2245.19)$ & $(5583.84,5867.85)$ \\
2 & {$[4,7]$} & $(13.724,13.819)$ & $(276.325,294.215)$ & $(1475.31,1691.15)$ & $(838.54,852.69)$ & $(2313.85,2541.84)$ \\
3 & {$[7,11]$} & $(13.836,14.145)$ & $(270.426,272.555)$ & $(1119.63,1323.17)$ & $(663.513,680.288)$ & $(1783.143,2003.458)$ \\
4 & {$[11,15]$} & $(13.027,15.633)$ & $(178.418,182.749)$ & $(638.244,652.872)$ & $(337.451,356.307)$ & $(975.695,100.179)$ \\
$\sum$ & & $(54.239,57.434)$ & $(1279.316,1321.83)$ & $(6728.304,7289.852)$ & $(3928.224,4134.475)$ & $(10656.528,11424.325)$ \\
\hline
\end{tabular}

Table 14. Numerical results by using the airfoil polynomials fuzzy collocation method; Case $2, d=2$.

\begin{tabular}{|c|c|c|c|c|c|c|}
\hline & {$\left[z_{k-1}^{*}, z_{k-1}^{*}\right]$} & $\left(\underline{x}_{2}^{*}, \bar{x}_{2}^{*}\right)$ & $\left(\underline{Q}\left(x_{2}^{*}\right), \bar{Q}\left(x_{2}^{*}\right)\right)$ & $\left(\underline{\pi}\left(x_{2}^{*}\right)_{R}, \bar{\pi}\left(x_{2}^{*}\right)_{R}\right)$ & $\left(\underline{\pi}\left(x_{2}^{*}\right)_{M}, \bar{\pi}\left(x_{2}^{*}\right)_{M}\right)$ & $\left(\underline{\pi}\left(x_{2}^{*}\right)_{C}, \bar{\pi}\left(x_{2}^{*}\right)_{C}\right)$ \\
\hline 1 & {$[0,3]$} & $(13.635,14.042)$ & $(417.12,436.38)$ & $(2744.22,2966.21)$ & $(1486.18,1613.44)$ & $(4230.4,4579.65)$ \\
\hline 2 & {$[3,8]$} & $(13.823,14.117)$ & $(475.276,490.81)$ & & & \\
\hline 3 & {$[8,12]$} & $(13.954,14.216)$ & $(239.055,241.667)$ & $(997.49,1107.48)$ & $(551.488,579.637)$ & $(1548.978$, \\
\hline 4 & {$[12,15]$} & $(13.932,14.347)$ & $(120.435,122.306)$ & $(447.627,460.803)$ & $(1977.312,2114.765)$ & $(2424.939,2575.568)$ \\
\hline$\Gamma$ & & $(55.344,56.722)$ & $(1251.877,1291.163)$ & $(6503.917,7112.603)$ & $(5432.27,5930.972)$ & $(11936.187,13043.575)$ \\
\hline
\end{tabular}

Table 15. Numerical results by using the airfoil polynomials fuzzy collocation method; Case $2, d=3$.

\begin{tabular}{|c|c|c|c|c|c|}
\hline$k^{*}\left[z_{k-1}^{*}, z_{k-1}^{*}\right]$ & $\left(\underline{x}_{3}^{*}, \bar{x}_{3}^{*}\right)$ & $\left(\underline{Q}\left(x_{3}^{*}\right), \bar{Q}\left(x_{3}^{*}\right)\right)$ & $\left(\underline{\pi}\left(x_{3}^{*}\right)_{R}, \bar{\pi}\left(x_{3}^{*}\right)_{R}\right)$ & $\left(\underline{\pi}\left(x_{3}^{*}\right)_{M}, \bar{\pi}\left(x_{3}^{*}\right)_{M}\right)$ & $\left(\underline{\pi}\left(x_{3}^{*}\right)_{C}, \bar{\pi}\left(x_{3}^{*}\right)_{C}\right)$ \\
\hline $1 \quad[0,4]$ & $(13.324,15.245)$ & $(514.033,534.014)$ & & & $(5244.55,5604.71)$ \\
\hline$[4,8]$ & & & & & \\
\hline$[8,15]$ & $(13.542,15.015)$ & $(361$. & & & \\
\hline$\sum$ & $(40.377,45.396)$ & $(1212.989,4412.831)$ & $(6388.05,6962.67)$ & $(3752.52,4077.31)$ & $(10140.57,11039.98)$ \\
\hline
\end{tabular}

Table 16. Numerical results by using the airfoil polynomials fuzzy collocation method; Case $3, d=1$.

\begin{tabular}{ccccccc}
\hline $\boldsymbol{k}^{*}\left[\boldsymbol{z}_{\boldsymbol{k}-\mathbf{1}}^{*}, \boldsymbol{z}_{\boldsymbol{k}-\mathbf{1}}^{*}\right]$ & $\left(\underline{\boldsymbol{x}}_{\mathbf{1}}^{*}, \overline{\boldsymbol{x}}_{\mathbf{1}}^{*}\right)$ & $\left(\underline{\boldsymbol{Q}}\left(\boldsymbol{x}_{\mathbf{1}}^{*}\right), \overline{\boldsymbol{Q}}\left(\boldsymbol{x}_{\mathbf{1}}^{*}\right)\right)$ & $\left(\underline{\boldsymbol{\pi}}\left(\boldsymbol{x}_{\mathbf{1}}^{*}\right)_{\boldsymbol{R}}, \overline{\boldsymbol{\pi}}\left(\boldsymbol{x}_{\mathbf{1}}^{*}\right)_{\boldsymbol{R}}\right)$ & $\left(\underline{\boldsymbol{\pi}}\left(\boldsymbol{x}_{\mathbf{1}}^{*}\right)_{M}, \overline{\boldsymbol{\pi}}\left(\boldsymbol{x}_{\mathbf{1}}^{*}\right)_{M}\right)$ & $\left(\underline{\boldsymbol{\pi}}\left(\boldsymbol{x}_{\mathbf{1}}^{*}\right)_{C}, \overline{\boldsymbol{\pi}}\left(\boldsymbol{x}_{\mathbf{1}}^{*}\right)_{\boldsymbol{C}}\right)$ \\
\hline 1 & {$[0,4]$} & $(13.619,13.843)$ & $(554.633,572.428)$ & $(3495.15,3622.82)$ & $(2088.28,2245.695)$ & $(5583.43,5868.515)$ \\
2 & {$[4,7]$} & $(13.725,13.826)$ & $(276.562,294.721)$ & $(1475.61,1691.84)$ & $(838.56,852.11)$ & $(2314.17,2543.95)$ \\
3 & {$[7,11]$} & $(13.814,14.239)$ & $(270.266,272.389)$ & $(1119.95,1323.84)$ & $(663.482,680.244)$ & $(1783.432,2004.084)$ \\
4 & {$[11,15]$} & $(13.156,15.699)$ & $(178.843,182.072)$ & $(638.422,652.517)$ & $(337.338,356.212)$ & $(975.76,1008.729)$ \\
$\sum$ & & $(54.314,57.607)$ & $(1280.304,1321.61)$ & $(6729.132,7291.017)$ & $(3927.66,4134.261)$ & $(10656.792,11425.278)$ \\
\hline
\end{tabular}


Table 17. Numerical results by using the airfoil polynomials fuzzy collocation method; Case $3, d=2$.

\begin{tabular}{ccccccc}
\hline $\boldsymbol{k}^{*}\left[\boldsymbol{z}_{\boldsymbol{k}-\mathbf{1}}^{*}, \boldsymbol{z}_{\boldsymbol{k}-\mathbf{1}}^{*}\right]$ & $\left(\underline{\boldsymbol{x}}_{\mathbf{2}}^{*}, \overline{\boldsymbol{x}}_{\mathbf{2}}^{*}\right)$ & $\left(\underline{\boldsymbol{Q}}\left(\boldsymbol{x}_{\mathbf{2}}^{*}\right), \overline{\boldsymbol{Q}}\left(\boldsymbol{x}_{\mathbf{2}}^{*}\right)\right)$ & $\left(\underline{\boldsymbol{\pi}}\left(\boldsymbol{x}_{\mathbf{2}}^{*}\right)_{\boldsymbol{R}}, \overline{\boldsymbol{\pi}}\left(\boldsymbol{x}_{\mathbf{2}}^{*}\right)_{\boldsymbol{R}}\right)$ & $\left(\underline{\boldsymbol{\pi}}\left(\boldsymbol{x}_{\mathbf{2}}^{*}\right)_{\boldsymbol{M}}, \overline{\boldsymbol{\pi}}\left(\boldsymbol{x}_{\mathbf{2}}^{*}\right)_{\boldsymbol{M}}\right)$ & $\left(\underline{\boldsymbol{\pi}}\left(\boldsymbol{x}_{\mathbf{2}}^{*}\right)_{C}, \overline{\boldsymbol{\pi}}\left(\boldsymbol{x}_{\mathbf{2}}^{*}\right)_{C}\right)$ \\
\hline 1 & {$[0,3]$} & $(13.609,14.025)$ & $(417.69,436.14)$ & $(2776.37,2966.52)$ & $(1486.15,1613.65)$ & $(4262.52,4580.17)$ \\
2 & {$[3,8]$} & $(13.813,14.171)$ & $(475.225,490.13)$ & $(2314.33,2578.43)$ & $(1417.43,1623.62)$ & $(3731.76,4202.05)$ \\
3 & {$[8,12]$} & $(13.942,14.217)$ & $(239.075,241.644)$ & $(997.77,1107.92)$ & $(551.475,579.616)$ & $(1549.245,1687.536)$ \\
4 & {$[12,15]$} & $(13.955,14.313)$ & $(120.416,122.308)$ & $(447.612,460.885)$ & $(1929.311,2114.747)$ & $(2376.923,2575.632)$ \\
$\sum$ & & $(55.319,56.726)$ & $(1252.406,1290.222)$ & $(6536.082,7113.752)$ & $(5384.366,5931.633)$ & $(11947.448,13045.385)$ \\
\hline
\end{tabular}

Table 18. Numerical results by using the airfoil polynomials fuzzy collocation method; Case $3, d=3$.

\begin{tabular}{ccccccc}
\hline $\boldsymbol{k}^{*}\left[\boldsymbol{z}_{\boldsymbol{k}-\mathbf{1}}^{*}, \boldsymbol{z}_{\boldsymbol{k}-\mathbf{1}}^{*}\right]$ & $\left(\underline{\boldsymbol{x}}_{\mathbf{3}}^{*}, \overline{\boldsymbol{x}}_{\mathbf{3}}^{*}\right)$ & $\left(\underline{\boldsymbol{Q}}\left(\boldsymbol{x}_{\mathbf{3}}^{*}\right), \overline{\boldsymbol{Q}}\left(\boldsymbol{x}_{\mathbf{3}}^{*}\right)\right)$ & $\left(\underline{\boldsymbol{\pi}}\left(\boldsymbol{x}_{\mathbf{3}}^{*}\right)_{\boldsymbol{R}}, \overline{\boldsymbol{\pi}}\left(\boldsymbol{x}_{\mathbf{3}}^{*}\right)_{\boldsymbol{R}}\right)$ & $\left(\underline{\boldsymbol{\pi}}\left(\boldsymbol{x}_{\mathbf{3}}^{*}\right)_{M}, \overline{\boldsymbol{\pi}}\left(\boldsymbol{x}_{\mathbf{3}}^{*}\right)_{\boldsymbol{M}}\right)$ & $\left(\underline{\boldsymbol{\pi}}\left(\boldsymbol{x}_{\mathbf{3}}^{*}\right)_{\boldsymbol{C}}, \overline{\boldsymbol{\pi}}\left(\boldsymbol{x}_{\mathbf{3}}^{*}\right)_{\boldsymbol{C}}\right)$ \\
\hline 1 & {$[0,4]$} & $(13.336,15.247)$ & $(514.037,534.054)$ & $(3325.35,3567.85)$ & $(1865.23,2037.78)$ & $(5190.58,5605.63)$ \\
2 & {$[4,8]$} & $(13.583,15.149)$ & $(337.812,3507.67)$ & $(1767.31,1955.46)$ & $(989.14,1124.27)$ & $(2756.45,3079.73)$ \\
3 & {$[8,15]$} & $(13.522,15.066)$ & $(361.165,371.213)$ & $(1285.99,1439.87)$ & $(897.17,915.89)$ & $(2183.16,2355.76)$ \\
$\sum$ & & $(40.441,45.489)$ & $(1213.014,4412.937)$ & $(6378.65,6963.18)$ & $(3742.54,4077.94)$ & $(10121.19,11041.12)$ \\
\hline
\end{tabular}

Table 19. Numerical results by using the fuzzy collocation method; Case $1, d=1$.

\begin{tabular}{ccccccc}
\hline $\boldsymbol{k}^{*}\left[\boldsymbol{z}_{\boldsymbol{k}-\mathbf{1}}^{*}, \boldsymbol{z}_{\boldsymbol{k}-\mathbf{1}}^{*}\right]$ & $\left(\underline{\boldsymbol{x}}_{\mathbf{1}}^{*}, \overline{\boldsymbol{x}}_{\mathbf{1}}^{*}\right)$ & $\left(\underline{\boldsymbol{Q}}\left(\boldsymbol{x}_{\mathbf{1}}^{*}\right), \overline{\boldsymbol{Q}}\left(\boldsymbol{x}_{\mathbf{1}}^{*}\right)\right)$ & $\left(\underline{\boldsymbol{\pi}}\left(\boldsymbol{x}_{\mathbf{1}}^{*}\right)_{\boldsymbol{R}}, \overline{\boldsymbol{\pi}}\left(\boldsymbol{x}_{\mathbf{1}}^{*}\right)_{\boldsymbol{R}}\right)$ & $\left(\underline{\boldsymbol{\pi}}\left(\boldsymbol{x}_{\mathbf{1}}^{*}\right)_{M}, \overline{\boldsymbol{\pi}}\left(\boldsymbol{x}_{\mathbf{1}}^{*}\right)_{M}\right)$ & $\left(\underline{\boldsymbol{\pi}}\left(\boldsymbol{x}_{\mathbf{1}}^{*}\right)_{C}, \overline{\boldsymbol{\pi}}\left(\boldsymbol{x}_{\mathbf{1}}^{*}\right)_{C}\right)$ \\
\hline 1 & {$[0,4]$} & $(13.623,13.843)$ & $(554.196,572.382)$ & $(3495.07,3622.13)$ & $(2088.43,2245.29)$ & $(5583.5,5867.42)$ \\
2 & {$[4,7]$} & $(13.765,13.822)$ & $(276.309,294.262)$ & $(1475.73,1691.26)$ & $(838.13,852.81)$ & $(2313.86,2544.07)$ \\
3 & {$[7,11]$} & $(13.877,14.032)$ & $(270.445,272.538)$ & $(1119.56,1323.88)$ & $(663.525,680.116)$ & $(1783.085,2003.996)$ \\
4 & {$[11,15]$} & $(13.086,15.653)$ & $(178.423,182.787)$ & $(638.234,652.866)$ & $(337.478,356.352)$ & $(975.712,1009.218)$ \\
$\sum$ & & $(54.351,57.35)$ & $(1279.373,1321.969)$ & $(6728.594,7290.136)$ & $(3927.563,4143.568)$ & $(10872.162)$ \\
\hline
\end{tabular}

Table 20. Numerical results by using the fuzzy collocation method; Case $1, d=2$.

\begin{tabular}{|c|c|c|c|c|c|c|}
\hline$k^{*}$ & {$\left[z_{k-1}^{*}, z_{k-1}^{*}\right]$} & $\left(\underline{x}_{2}^{*}, \bar{x}_{2}^{*}\right)$ & $\left(\underline{Q}\left(x_{2}^{*}\right), \bar{Q}\left(x_{2}^{*}\right)\right)$ & $\left(\underline{\pi}\left(x_{2}^{*}\right)_{R}, \bar{\pi}\left(x_{2}^{*}\right)_{R}\right)$ & $\left(\underline{\pi}\left(x_{2}^{*}\right)_{M}, \bar{\pi}\left(x_{2}^{*}\right)_{M}\right)$ & $\left(\underline{\pi}\left(x_{2}^{*}\right)_{C}, \bar{\pi}\left(x_{2}^{*}\right)_{C}\right)$ \\
\hline 1 & {$[0,3]$} & $(13.698,14.032)$ & $(417.21,436.77)$ & $(2744.26,2966.35)$ & $(1486.33,1613.21)$ & $(4230.59,4589.56)$ \\
\hline 2 & {$[3,8]$} & $(13.818,14.151)$ & $(475.235,490.56)$ & $(2314.68,2578.65)$ & $(1417.19,1623.48)$ & $(3731.87,4202.13)$ \\
\hline 3 & {$[8,12]$} & $(13.904,14.266)$ & $(239.093,241.687)$ & $(997.13,1107.44)$ & $(551.426,579.605)$ & $(1548.556,1687.045)$ \\
\hline 4 & {$[12,15]$} & $(13.927,14.387)$ & $(120.408,122.312)$ & $(447.625,460.879)$ & $(1977.337,2114.755)$ & $(2424.962,2575.634)$ \\
\hline$\sum$ & & $(55.347,56.836)$ & $(1251.946,1291.329)$ & $(6503.695,7113.319)$ & $(5432.283,5931.05)$ & $(11935.978,13006.369)$ \\
\hline
\end{tabular}

Table 21. Numerical results by using the fuzzy collocation method; Case $1, d=3$.

\begin{tabular}{ccccccc}
\hline $\boldsymbol{k}^{*}\left[\boldsymbol{z}_{\boldsymbol{k}-\mathbf{1}}^{*}, \boldsymbol{z}_{\boldsymbol{k}-\mathbf{1}}^{*}\right]$ & $\left(\underline{\boldsymbol{x}}_{\mathbf{3}}^{*}, \overline{\boldsymbol{x}}_{\mathbf{3}}^{*}\right)$ & $\left(\underline{\boldsymbol{Q}}\left(\boldsymbol{x}_{\mathbf{3}}^{*}\right), \overline{\boldsymbol{Q}}\left(\boldsymbol{x}_{\mathbf{3}}^{*}\right)\right)$ & $\left(\underline{\boldsymbol{\pi}}\left(\boldsymbol{x}_{\mathbf{3}}^{*}\right)_{\boldsymbol{R}}, \overline{\boldsymbol{\pi}}\left(\boldsymbol{x}_{\mathbf{3}}^{*}\right)_{\boldsymbol{R}}\right)$ & $\left(\underline{\boldsymbol{\pi}}\left(\boldsymbol{x}_{\mathbf{3}}^{*}\right)_{\boldsymbol{M}}, \overline{\boldsymbol{\pi}}\left(\boldsymbol{x}_{\mathbf{3}}^{*}\right)_{\boldsymbol{M}}\right)$ & $\left(\underline{\boldsymbol{\pi}}\left(\boldsymbol{x}_{\mathbf{3}}^{*}\right)_{\boldsymbol{C}}, \overline{\boldsymbol{\pi}}\left(\boldsymbol{x}_{\mathbf{3}}^{*}\right)_{\boldsymbol{C}}\right)$ \\
\hline 1 & {$[0,4]$} & $(13.307,15.267)$ & $(514.027,534.043)$ & $(3342.09,3567.17)$ & $(1865.48,2037.22)$ & $(5207.57,5604.39)$ \\
2 & {$[4,8]$} & $(13.515,15.182)$ & $(337.875,3507.45)$ & $(1733.78,1955.39)$ & $(989.05,1124.73)$ & $(2722.83,3080.12)$ \\
3 & {$[8,15]$} & $(13.513,15.088)$ & $(361.179,371.257)$ & $(1276.14,1439.49)$ & $(897.44,915.69)$ & $(2173.58,2355.18)$ \\
$\sum$ & & $(40.335,45.537)$ & $(1213.081,4412.75)$ & $(6352.01,6962.05)$ & $(3751.97,4077.64)$ & $(10103.98,11039.69)$ \\
\hline
\end{tabular}

Table 22. Numerical results by using the fuzzy collocation method; Case $2, d=1$.

\begin{tabular}{ccccccc}
\hline $\boldsymbol{k}^{*}\left[\boldsymbol{z}_{\boldsymbol{k}-\mathbf{1}}^{*}, \boldsymbol{z}_{k-\mathbf{1}}^{*}\right]$ & $\left(\underline{\boldsymbol{x}}_{\mathbf{1}}^{*}, \overline{\boldsymbol{x}}_{\mathbf{1}}^{*}\right)$ & $\left(\underline{\boldsymbol{Q}}\left(\boldsymbol{x}_{\mathbf{1}}^{*}\right), \overline{\boldsymbol{Q}}\left(\boldsymbol{x}_{\mathbf{1}}^{*}\right)\right)$ & $\left(\underline{\boldsymbol{\pi}}\left(\boldsymbol{x}_{\mathbf{1}}^{*}\right)_{\boldsymbol{R}}, \overline{\boldsymbol{\pi}}\left(\boldsymbol{x}_{\mathbf{1}}^{*}\right)_{\boldsymbol{R}}\right)$ & $\left(\underline{\boldsymbol{\pi}}\left(\boldsymbol{x}_{\mathbf{1}}^{*}\right)_{\boldsymbol{M}}, \overline{\boldsymbol{\pi}}\left(\boldsymbol{x}_{\mathbf{1}}^{*}\right)_{\boldsymbol{M}}\right)$ & $\left(\underline{\boldsymbol{\pi}}\left(\boldsymbol{x}_{\mathbf{1}}^{*}\right)_{\boldsymbol{C}}, \overline{\boldsymbol{\pi}}\left(\boldsymbol{x}_{\mathbf{1}}^{*}\right)_{C}\right)$ \\
\hline 1 & {$[0,4]$} & $(13.676,13.832)$ & $(554.167,572.315)$ & $(3495.76,3622.08)$ & $(2073.72,2245.27)$ & $(5569.48,5867.35)$ \\
2 & {$[4,7]$} & $(13.719,13.854)$ & $(276.333,294.289)$ & $(1475.23,1691.48)$ & $(838.82,852.56)$ & $(2314.05,2544.04)$ \\
3 & {$[7,11]$} & $(13.826,14.119)$ & $(270.435,272.581)$ & $(1119.51,1323.33)$ & $(663.563,680.297)$ & $(1783.073,2003.627)$ \\
4 & {$[11,15]$} & $(13.013,15.677)$ & $(178.449,182.723)$ & $(638.225,652.817)$ & $(337.428,356.391)$ & $(975.653,1009.208)$ \\
$\Sigma$ & & $(54.234,57.482)$ & $(1279.384,1321.908)$ & $(6728.725,7289.707)$ & $(3913.531,4134.518)$ & $(10642.256,11424.225)$ \\
\hline
\end{tabular}


Table 23. Numerical results by using the fuzzy collocation method; Case $2, d=2$.

\begin{tabular}{ccccccc}
\hline $\boldsymbol{k}^{*}\left[\boldsymbol{z}_{\boldsymbol{k}-\mathbf{1}}^{*}, \boldsymbol{z}_{\boldsymbol{k}-\mathbf{1}}^{*}\right]$ & $\left(\underline{\boldsymbol{x}}_{\mathbf{2}}^{*}, \overline{\boldsymbol{x}}_{\mathbf{2}}^{*}\right)$ & $\left(\underline{\boldsymbol{Q}}\left(\boldsymbol{x}_{\mathbf{2}}^{*}\right), \overline{\boldsymbol{Q}}\left(\boldsymbol{x}_{\mathbf{2}}^{*}\right)\right)$ & $\left(\underline{\boldsymbol{\pi}}\left(\boldsymbol{x}_{\mathbf{2}}^{*}\right)_{\boldsymbol{R}}, \overline{\boldsymbol{\pi}}\left(\boldsymbol{x}_{\mathbf{2}}^{*}\right)_{\boldsymbol{R}}\right)$ & $\left(\underline{\boldsymbol{\pi}}\left(\boldsymbol{x}_{\mathbf{2}}^{*}\right)_{M}, \overline{\boldsymbol{\pi}}\left(\boldsymbol{x}_{\mathbf{2}}^{*}\right)_{\boldsymbol{M}}\right)$ & $\left(\underline{\boldsymbol{\pi}}\left(\boldsymbol{x}_{\mathbf{2}}^{*}\right)_{C}, \overline{\boldsymbol{\pi}}\left(\boldsymbol{x}_{\mathbf{2}}^{*}\right)_{C}\right)$ \\
\hline 1 & {$[0,3]$} & $(13.643,14.065)$ & $(417.27,436.89)$ & $(2744.49,2966.17)$ & $(1486.18,1613.74)$ & $(4230.67,4579.91)$ \\
2 & {$[3,8]$} & $(13.814,14.133)$ & $(475.206,490.35)$ & $(2314.67,2578.19)$ & $(1417.37,1623.59)$ & $(3732.04,4201.78)$ \\
3 & {$[8,12]$} & $(13.971,14.224)$ & $(239.078,241.615)$ & $(997.76,1107.83)$ & $(551.485,579.642)$ & $(1549.242,1687.472)$ \\
4 & {$[12,15]$} & $(13.977,14.328)$ & $(120.427,122.358)$ & $(447.623,460.803)$ & $(1977.355,2114.758)$ & $(2424.978,2575.561)$ \\
$\sum$ & & $(55.405,56.75)$ & $(1251.981,1291.213)$ & $(6504.543,7112.993)$ & $(5432.39,5931.73)$ & $(11936.933,13044.723)$ \\
\hline
\end{tabular}

Table 24. Numerical results by using the fuzzy collocation method; Case $2, d=3$.

\begin{tabular}{ccccccc}
\hline $\boldsymbol{k}^{*}\left[\boldsymbol{z}_{\boldsymbol{k}-\mathbf{1}}^{*}, \boldsymbol{z}_{\boldsymbol{k}-\mathbf{1}}^{*}\right]$ & $\left(\underline{\left.\boldsymbol{x}_{\mathbf{3}}^{*}, \overline{\boldsymbol{x}}_{\mathbf{3}}^{*}\right)}\right.$ & $\left(\underline{\boldsymbol{Q}}\left(\boldsymbol{x}_{\mathbf{3}}^{*}\right), \overline{\boldsymbol{Q}}\left(\boldsymbol{x}_{\mathbf{3}}^{*}\right)\right)$ & $\left(\underline{\boldsymbol{\pi}}\left(\boldsymbol{x}_{\mathbf{3}}^{*}\right)_{\boldsymbol{R}}, \overline{\boldsymbol{\pi}}\left(\boldsymbol{x}_{\mathbf{3}}^{*}\right)_{\boldsymbol{R}}\right)$ & $\left(\underline{\boldsymbol{\pi}}\left(\boldsymbol{x}_{\mathbf{3}}^{*}\right)_{\boldsymbol{M}}, \overline{\boldsymbol{\pi}}\left(\boldsymbol{x}_{\mathbf{3}}^{*}\right)_{\boldsymbol{M}}\right)$ & $\left(\underline{\boldsymbol{\pi}}\left(\boldsymbol{x}_{\mathbf{3}}^{*}\right)_{\boldsymbol{C}}, \overline{\boldsymbol{\pi}}\left(\boldsymbol{x}_{\mathbf{3}}^{*}\right)_{\boldsymbol{C}}\right)$ \\
\hline 1 & {$[0,4]$} & $(13.316,15.289)$ & $(514.077,534.065)$ & $(3378.22,3567.58)$ & $(1865.91,2037.49)$ & $(5244.13,5605.07)$ \\
2 & {$[4,8]$} & $(13.525,15.156)$ & $(337.828,3507.13)$ & $(1733.43,1955.36)$ & $(989.66,1124.92)$ & $(2723.09,3080.28)$ \\
3 & {$[8,15]$} & $(13.584,15.036)$ & $(361.192,371.271)$ & $(1276.55,1439.77)$ & $(897.43,915.51)$ & $(2173.98,2355.28)$ \\
$\sum$ & & $(40.425,45.481)$ & $(1213.097,4412.466)$ & $(6378.2,6962.71)$ & $(3753,4077.92)$ & $(10141.2,11040.63)$ \\
\hline
\end{tabular}

\section{Algorithm:}

Step 1. Set $n \leftarrow 0$;

Step 2. Solve the systems (12), (15), (18), (28), (31), (34), (41), (46);

Step 3. If $D\left(\widetilde{u}_{n+1}\left(x_{d}, t\right), \widetilde{u}_{n}\left(x_{d}, t\right)\right)<\epsilon$, then go to step 4, else, $n \leftarrow n+1$ and go to Step 2;

Step 4. Print $\widetilde{u}_{n}\left(x_{d}, t\right)$ as the approximation of the exact solution.

With respect to the type of derivative that was defined in Eq. (7) and generated system in Eqs. (15) and (16), numerical results and amounts of errors in $x_{1}, x_{2}, x_{3}$ can be obtained as follows:

$$
\begin{aligned}
& D\left(\widetilde{E}_{n}\left(x_{1}, t\right), \widetilde{0}\right) \leq 0.000286, \\
& D\left(\widetilde{E}_{n}\left(x_{2}, t\right), \widetilde{0}\right) \leq 0.000278, \\
& D\left(\widetilde{E}_{n}\left(x_{3}, t\right), \widetilde{0}\right) \leq 0.000269 .
\end{aligned}
$$

With respect to the type of derivative that was defined in Eq. (8) and generated system in Eqs. (18) and (19), numerical results and amounts of errors in $x_{1}, x_{2}, x_{3}$ can be obtained as follows:

$$
\begin{aligned}
& D\left(\widetilde{E}_{n}\left(x_{1}, t\right), \widetilde{0}\right) \leq 0.000249, \\
& D\left(\widetilde{E}_{n}\left(x_{2}, t\right), \widetilde{0}\right) \leq 0.000237, \\
& D\left(\widetilde{E}_{n}\left(x_{3}, t\right), \widetilde{0}\right) \leq 0.000227 .
\end{aligned}
$$

With respect to the type of derivative that was defined in Eq. (9) and generated system in Eqs. (21) and (22), numerical results and amounts of errors in $x_{1}, x_{2}, x_{3}$ can be obtained as follows:

$$
\begin{aligned}
& D\left(\widetilde{E}_{n}\left(x_{1}, t\right), \widetilde{0}\right) \leq 0.000238, \\
& D\left(\widetilde{E}_{n}\left(x_{2}, t\right), \widetilde{0}\right) \leq 0.000229, \\
& D\left(\widetilde{E}_{n}\left(x_{3}, t\right), \widetilde{0}\right) \leq 0.000221 .
\end{aligned}
$$

With respect to the type of derivative that was defined in Eq. (23) and generated system in Eqs. (31) and (32), numerical results and amounts of errors in $x_{1}, x_{2}, x_{3}$ can be obtained as follows:

$$
\begin{aligned}
& D\left(\widetilde{E}_{n}\left(x_{1}, t\right), \widetilde{0}\right) \leq 0.000263, \\
& D\left(\widetilde{E}_{n}\left(x_{2}, t\right), \widetilde{0}\right) \leq 0.000254, \\
& D\left(\widetilde{E}_{n}\left(x_{3}, t\right), \widetilde{0}\right) \leq 0.000241 .
\end{aligned}
$$

With respect to the type of derivative that was defined in Eq. (24) and generated system in Eqs. (34) and (35), numerical results and amounts of errors in $x_{1}, x_{2}, x_{3}$ can be obtained as follows:

$$
\begin{aligned}
& D\left(\widetilde{E}_{n}\left(x_{1}, t\right), \widetilde{0}\right) \leq 0.000225, \\
& D\left(\widetilde{E}_{n}\left(x_{2}, t\right), \widetilde{0}\right) \leq 0.000218, \\
& D\left(\widetilde{E}_{n}\left(x_{3}, t\right), \widetilde{0}\right) \leq 0.000209 .
\end{aligned}
$$


With respect to the type of derivative that was defined in Eq. (25) and generated system in Eqs. (37) and (38), numerical results and amounts of errors in $x_{1}, x_{2}, x_{3}$ can be obtained as follows:

$$
\begin{aligned}
& D\left(\widetilde{E}_{n}\left(x_{1}, t\right), \widetilde{0}\right) \leq 0.000219, \\
& D\left(\widetilde{E}_{n}\left(x_{2}, t\right), \widetilde{0}\right) \leq 0.000205, \\
& D\left(\widetilde{E}_{n}\left(x_{3}, t\right), \widetilde{0}\right) \leq 0.000208 .
\end{aligned}
$$

With respect to the type of derivative of $\sum_{i=0}^{n} \widetilde{a_{i}} h_{i}\left(x_{d}\right) h_{i}(t)$ that is $(i)$-differentiable and by solving the generated system in Eqs. (44) and (45), numerical results and amounts of errors in $x_{1}, x_{2}, x_{3}$ can be obtained as follows:

$$
\begin{aligned}
& D\left(\widetilde{E}_{n}\left(x_{1}, t\right), \widetilde{0}\right) \leq 0.000259, \\
& D\left(\widetilde{E}_{n}\left(x_{2}, t\right), \widetilde{0}\right) \leq 0.000248, \\
& D\left(\widetilde{E}_{n}\left(x_{3}, t\right), \widetilde{0}\right) \leq 0.000239 .
\end{aligned}
$$

With respect to the type of derivative of $\sum_{i=0}^{n} \widetilde{a_{i}} h_{i}\left(x_{d}\right) h_{i}(t)$ that is $(i i)$-differentiable, and by solving the generated system in Eqs. (49) and (50), numerical results and amounts of errors in $x_{1}, x_{2}, x_{3}$ can be obtained as follows:

$$
\begin{aligned}
& D\left(\widetilde{E}_{n}\left(x_{1}, t\right), \widetilde{0}\right) \leq 0.000228, \\
& D\left(\widetilde{E}_{n}\left(x_{2}, t\right), \widetilde{0}\right) \leq 0.000223, \\
& D\left(\widetilde{E}_{n}\left(x_{3}, t\right), \widetilde{0}\right) \leq 0.000216 .
\end{aligned}
$$

Based on the obtained results, we can conclude that the error and number of iterations in the Jacobi polynomials method are less than those in the airfoil polynomials method and fuzzy collocation method. Moreover, for better understanding, the obtained results in these algorithms have been plotted on the graphs in Figures 1-3.

\section{Conclusion}

The contributions of the current study are twofold. First, it proposes a fuzzy multi-product dynamic pricing and inventory policies over a multi-period planning horizon with deteriorating products in continuous time.

Second, it proposes three novel fuzzy expansion methods including Jacobi polynomials, airfoil polynomials, and fuzzy collocation methods for solving the proposed model. Accordingly, it is concluded

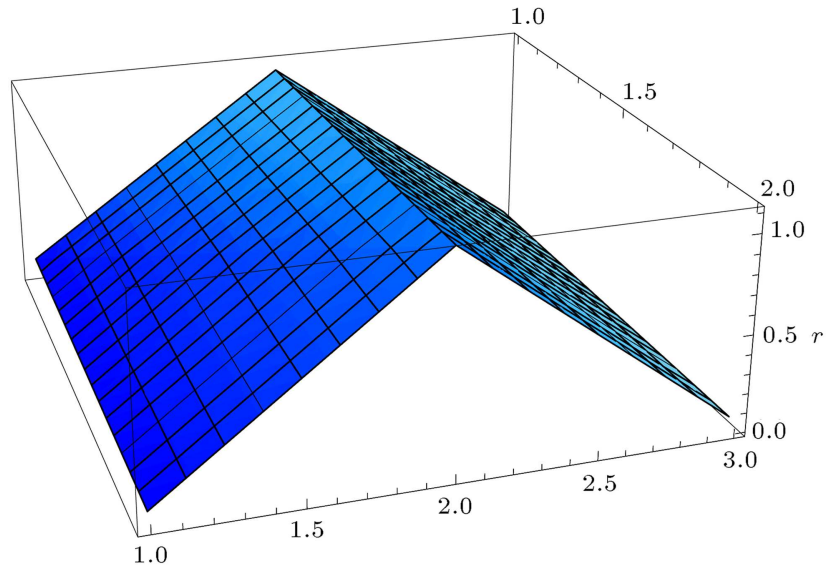

Figure 1. The obtained discount profit by Jacobi fuzzy collocation method, $d=1$.

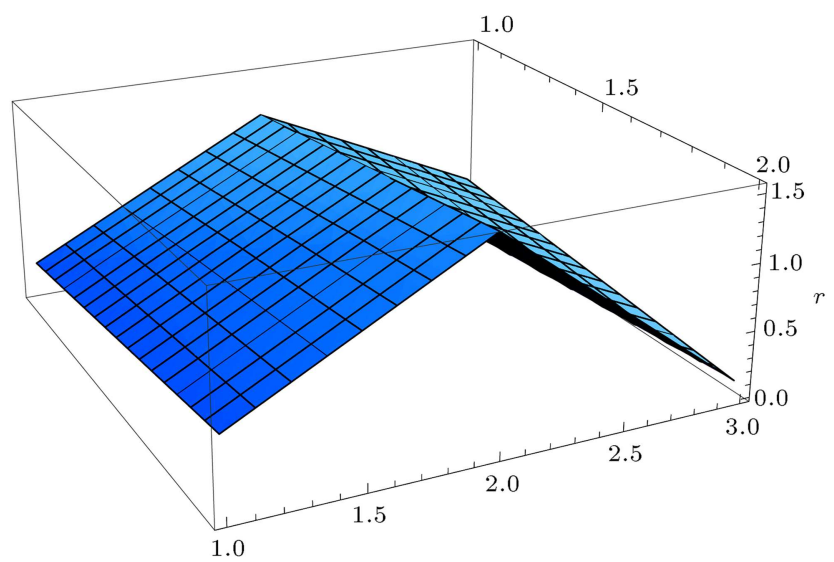

Figure 2. The obtained discount profit by airfoil fuzzy collocation method, $d=1$.

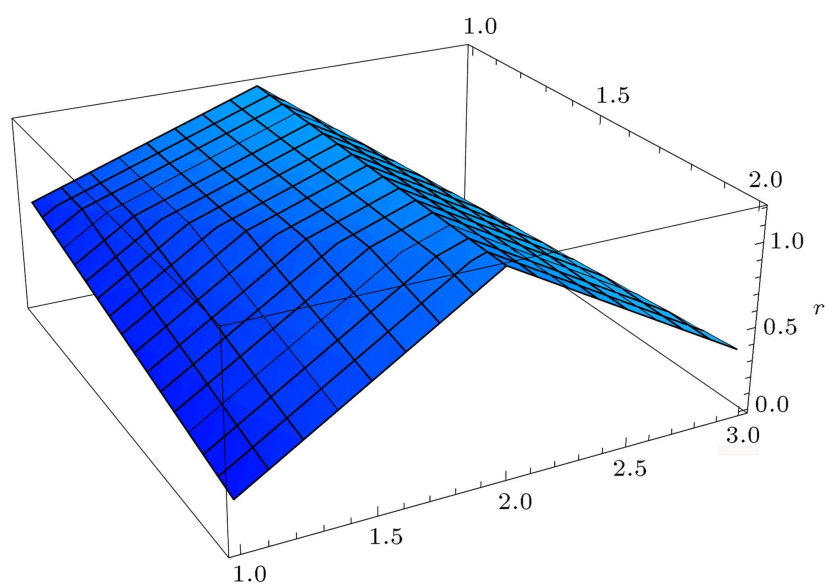

Figure 3. The obtained discount profit by fuzzy collocation method, $d=1$.

that the error and number of iterations in the Jacobi polynomials method are less than those in the airfoil polynomials method and fuzzy collocation method. For future inquiries, it is suggested to apply the proposed solution approaches in other pricing and inventory models with various assumptions. 


\section{References}

1. Pang, Z. "Optimal dynamic pricing and inventory control with stock deterioration and partial backordering", Operations Research Letters, 39(5), pp. 375-379 (2011).

2. Samadi, F., Mirzazadeh, A. and Pedram, M.M. "Fuzzy pricing, marketing and service planning in a fuzzy inventory model: A geometric programming approach", Applied Mathematical Modelling, 37(10), pp. 66836694 (2013).

3. Negoita, C., Zadeh, L. and Zimmermann, H. "Fuzzy sets as a basis for a theory of possibility", Fuzzy Sets and Systems, 1, pp. 3-28 (1978).

4. Jrgensen, S. and Kort, P.M. "Optimal pricing and inventory policies: Centralized and decentralized decision making", European Journal of Operational Research, 138(3), pp. 578-600 (2002).

5. Gupta, D., Hill, A.V. and Bouzdine-Chameeva, T. "A pricing model for clearing end-of-season retail inventory", European Journal of Operational Research, 170(2), pp. 518-540 (2006).

6. Jung, H. and Klein, C.M. "Optimal inventory policies for profit maximizing EOQ models under various cost functions", European Journal of Operational Research, 174(2), pp. 689-705 (2006).

7. Pal, P., Bhunia, A.K. and Goyal, S.K. "On optimal partially integrated production and marketing policy with variable demand under flexibility and reliability considerations via genetic algorithm", Applied Mathematics and Computation, 188(1), pp. 525-537 (2007).

8. Transchel, S. and Minner, S. "The impact of dynamic pricing on the economic order decision", European Journal of Operational Research, 198(3), pp. 773-789 (2009).

9. Herbon, Avi, Eugene Levner, and Cheng, T.C.E. "Perishable inventory management with dynamic pricing using time-temperature indicators linked to automatic detecting devices", International Journal of Production Economics, 147, pp. 605-613 (2014).

10. Chen, L.T. "Optimal dynamic policies for integratedproduction and marketing planning in businessto-business marketplaces", International Journal of Production Economics, 153, pp. 46-53 (2014).

11. Liu, S.T. "Fuzzy profit measures for a fuzzy economic order quantity model", Applied Mathematical Modelling, 32(10), pp. 2076-2086 (2008).

12. Sadjadi, S.J., Ghazanfari, M. and Yousefli, A, "Fuzzy pricing and marketing planning model: A possibilistic geometric programming approach", Expert Systems with Applications, 37(4), pp. 3392-3397 (2010).
13. Soni, H.N. and Joshi. M. "A fuzzy framework for coordinating pricing and inventory policies for deteriorating items under retailer partial trade credit financing", Computers and Industrial Engineering, 66(4), pp. 865878 (2013).

14. Coşgun, Ö, Ekinci, Y and Yanık, S. "Fuzzy rulebaseddemand forecasting for dynamic pricing of a maritime company", Knowledge-Based Systems, 70, pp. 88-96 (2014).

15. Zhao, J. and Wang, L. "Pricing and retail service decisions in fuzzy uncertainty environments", $A p$ plied Mathematics and Computation, 250, pp. 580-592 (2015).

16. Sadeghi, J. and Akhavan Niaki, S.T. "Two parameter tuned multi-objective evolutionary algorithms for a bi-objective vendor managed inventory model with trapezoidal fuzzy demand", Applied Soft Computing, 30, pp. 567-576 (2015).

17. Bede, Barnabas, and Sorin G. Gal. "Generalizations of the differentiability of fuzzy-number-valued functions with applications to fuzzy differential equations", Fuzzy Sets and Systems, 151(3), pp. 581-599 (2005).

18. Chalco-Cano, Y. and Román-Flores, H. "On new solutions offuzzy differential equations", Chaos, Solitons and Fractals, 38(1), pp. 112-119 (2008).

19. Li, Xianjuan, and Tao Tang. "Convergence analysis of Jacobi spectral collocation methods for Abel-Volterra integral equations of second kind", Frontiers of Mathematics in China, 7(1), pp. 69-84 (2012).

20. Desmarais, R.N. and Bland, S.R. "Tables of properties of airfoil polynomials", National Aeronautics and Space Administration, Langley Research Center 1343 (1995).

21. Behzadi, Sh.S. "A new study on first-order fuzzy Fredholm-Volterra integro-differential equations by Jacobi polynomials and collocation methods", Soft Computing, 19(2), pp. 421-429 (2015).

\section{Biographies}

Behnam Vahdani received $\mathrm{PhD}$ degree from the Department of Industrial Engineering at University of Tehran. He also obtained BS and MS degrees in Industrial Engineering. He is the member of Iran's National Elites Foundation. His current research interests include supply chain network design, facility location and design, logistics planning and scheduling, multicriteria decision making, uncertain programming, artificial neural networks, meta-heuristics algorithms, and operations research applications. He has published several papers and book chapters in the aforementioned areas.

Shadan Sadigh Behzadi was born in Tehran, Iran, in 1983. She received BSc and MSc degrees in Applied Mathematics, with the specialty of numerical 
analysis, from Islamic Azad University, Central Tehran Branch, and PHD degree in Applied Mathematics, with the specialty of numerical analysis, from Science and Research Branch, Islamic Azad University. Her main research interests include numerical solution of nonlinear Fredholm-Volterra integro-differential equation systems, ordinary differential equation, partial differential equation, fuzzy system, and some equations in fluid dynamics.

Tofigh Allahviranloo was born in Khoy in West Azerbaijan Province. He received PhD degree in 2001. Now, he is a professor of applied mathematics. He has published more than 130 papers in international journals and is Editor in Chief and Associated Editor in more than 5 journals. 